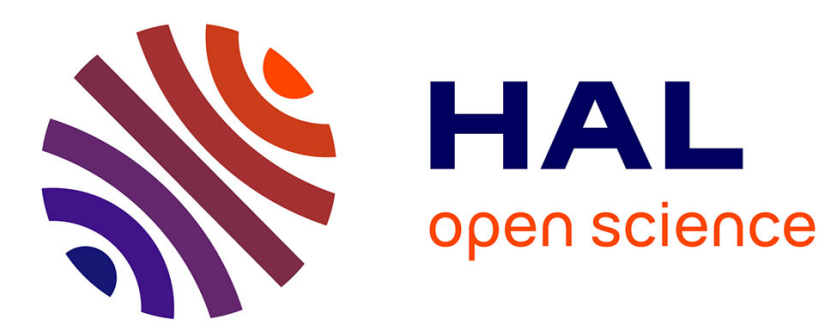

\title{
Lecture notes on rational polytopes and finite generation Mihai Päun
}

\section{To cite this version:}

Mihai Päun. Lecture notes on rational polytopes and finite generation. 2010. hal-00452374

\section{HAL Id: hal-00452374 \\ https://hal.science/hal-00452374}

Preprint submitted on 2 Feb 2010

HAL is a multi-disciplinary open access archive for the deposit and dissemination of scientific research documents, whether they are published or not. The documents may come from teaching and research institutions in France or abroad, or from public or private research centers.
L'archive ouverte pluridisciplinaire HAL, est destinée au dépôt et à la diffusion de documents scientifiques de niveau recherche, publiés ou non, émanant des établissements d'enseignement et de recherche français ou étrangers, des laboratoires publics ou privés. 


\title{
Lecture notes on rational polytopes and finite generation
}

\author{
Mihai Păun \\ Institut Élie Cartan, Nancy \\ and \\ Korea Institute for Advanced Study, Seoul
}

\section{$\S 0$ Introduction}

Let $X$ be a non-singular, projective manifold, and let $\left(Y_{j}\right)_{j=1, \ldots, N}$ be a set of nonsingular hypersurfaces of $X$, having strictly normal crossings. We denote by $A \rightarrow X$ an ample $\mathbb{Q}$-bundle on $X$. Following V. Shokurov, (cf. [24]) we define the set

$$
\mathcal{E}_{Y, A}:=\left\{\tau \in[0,1]^{N}: K_{X}+Y_{\tau}+A \in \operatorname{Psef}(X)\right\}
$$

where $Y_{\tau}:=\sum_{j=1}^{N} \tau^{j} Y_{j}$.

Let $\mathcal{L} \subset \mathbb{R}^{r}$ be an $r$-dimensional polytope, whose vertices have rational coordinates (i.e. a rational polytope). For $j=1, . ., N$, let $l^{j}: \mathbb{R}^{r} \rightarrow \mathbb{R}$ be a set of affine forms defined over $\mathbb{Q}$, such that

$$
0 \leq l^{j}(\theta) \leq 1-\varepsilon_{0}
$$

for all $\theta \in \mathcal{L}$, where $\varepsilon_{0}$ is a positive real number.

We denote by $d:=\left(d^{0}, \ldots, d^{r}\right)$ an element of $\mathbb{Z}^{r+1}$, and we introduce the set

$$
\Gamma_{d}:=\left\{(m, \theta) \in \mathbb{Z}_{+} \times \mathcal{L}: \forall j=0, \ldots, r, \quad m \theta^{j} \in d^{j} \mathbb{Z}, \quad l(\theta) \in \mathcal{E}_{Y, A}\right\}
$$

where by convention we put $\theta^{0}:=1$ in the relations above.

Our focus in this article will be on the following ring of holomorphic sections

$$
\mathcal{A}_{r}(X):=\bigoplus_{(m, \theta) \in \Gamma_{d}} H^{0}\left(X, m\left(K_{X}+\sum_{j=1}^{N} l^{j}(\theta) Y_{j}+A\right)\right)
$$

where we assume that the coordinates of $d$ are divisible enough.

In the framework of the minimal models program, the following result was established by C. Birkar, P. Cascini, C. Hacon and J. McKernan in [2] (one can profitably consult the enlightening presentations in [10], [15] of this article).

0.1 Theorem[2]. The following assertions are true.

(i) The set $\mathcal{E}_{Y, A}$ is a rational polytope ;

(ii) The ring $\mathcal{A}_{r}(X)$ defined above is finitely generated. 
A first remark about 0.1 is that the properties of the set $\mathcal{E}_{Y, A}$ are crucial for the original proof given in [2]. Concerning the finite generation part of the theorem 0.1, we mention the very elegant approach due to Y.-T. Siu (see [28], [29], [30]), which does not use V. Shokurov polytopes technique. Instead, he introduces new invariants associated to linear series (the "minimal discrepancy subspace"), which most certainly will be useful in other contexts.

As the title of the present article tries to suggest, our original contribution in this paper is somehow very modest : we propose a proof of the theorem 0.1 guided by the same principles as in [2], [19] and [28]. In spirit, we follow to a large extent the article [19] ; however, there are some important parts of the arguments in this article which we were not able to understand, so the main technical aspects of our approach are necessarily different (see the sections 2.C and 3 for more comments in this respect). One significant difference is that the core of our arguments relies exclusively on the extension theorems established in [22]. The theory of closed positive currents and its relationship with the algebraic geometry as developed by J.-P. Demailly and Y.-T. Siu over many years plays an important rôle in this text. Needless to say that the original proof [2] does not use such tools, and we do not claim at all that they are indispensable ; we just hope that the flexibility of the techniques employed here may be suitable for forthcoming research around these topics.

Along the following lines, we try to motivate the formulation of the point ii) above (and to highlight some of the important steps of a particular case of its proof). A first intuitive reason is that in this way we take into account a consistent part of statements of the inductive process in the original article [2], but here it is a more "down to Earth" explanation.

Let $L:=\sum_{j=1}^{N} \nu^{j} Y_{j}+A$ be a $\mathbb{Q}$-line bundle, where $0<\nu^{j}<1$, and let us say that we are willing to prove the finite generation of the algebra

$$
\mathcal{R}:=\bigoplus_{m \in d Z} H^{0}\left(X, m\left(K_{X}+L\right)\right)
$$

where $d$ is a divisible enough integer. Let $\varphi_{m_{0}}$ be a metric on $K_{X}+L$ induced by the linear system $\left|m_{0}\left(K_{X}+L\right)\right|$, and let $\varphi_{\text {min }}$ be the metric with minimal singularities on the same bundle, induced by the all the elements of $\mathcal{R}$, (see [7], [9], [26]).

If these two metrics are equivalent i.e. if $\left|\varphi_{\text {min }}-\varphi_{m_{0}}\right|=\mathcal{O}(1)$ on $X$, then the result we seek is obvious.

If not, then we can use $\varphi_{m_{0}}$ as an incomplete linear system and apply a slightly modified version of the $x$-method as in ([2], [22]) ; the outcome is that the finite generation of $\mathcal{R}$ is reduced to the finite generation of the algebra

$$
\mathcal{R}^{\prime}:=\bigoplus_{m \in d Z} H^{0}\left(X, m\left(K_{X}+S+L^{\prime}\right)\right)
$$

where $L^{\prime}=\sum_{j=1}^{K} \mu^{j} Y_{j}^{\prime}+A$ is a $\mathbb{Q}$-line bundle with $0<\mu^{j}<1$, the hypersurfaces $\left(S, Y_{j}^{\prime}\right)$ have strictly normal crossings and $\left(Y_{j}^{\prime}\right)$ are mutually disjoint. Moreover, we can assume the existence of an element of $\mathcal{R}^{\prime}$ whose restriction to $S$ is not identically zero. 
Then the restricted algebra

$$
\mathcal{R}_{\mid S}^{\prime}:=\operatorname{Im}\left(\bigoplus_{m \in d Z} H^{0}\left(X, m\left(K_{X}+S+L^{\prime}\right)\right) \rightarrow \bigoplus_{m \in d Z} H^{0}\left(S, m\left(K_{S}+L_{\mid S}^{\prime}\right)\right)\right)
$$

is easily identified via the extension of pluricanonical forms results, cf. [1], [14] as follows. There exists an effective $\mathbb{Q}$-divisor $\Delta_{S}:=\sum_{j=1}^{K} \rho^{j} Y_{j \mid S}^{\prime}$ on $S$, such that : a section of $m\left(K_{S}+L_{\mid S}^{\prime}\right)$ extends to $X$ if and only if its zeroes divisor is greater than $m \sum_{j} \min \left(\rho^{j}, \nu^{j}\right) Y_{j \mid S}^{\prime}$

The existence of $\Delta_{S}$ together with the induction hypothesis shows that $\mathcal{R}_{\mid S}^{\prime}$ is finitely generated ; in analytic terms, this implies that the restriction $\varphi_{\min \mid \mathrm{S}}$ has logarithmic poles. In order to derive a similar conclusion locally near $S$, we have to understand the "normal directions", i.e. the restricted algebra

$$
\operatorname{Im}\left(\bigoplus_{m, k} H^{0}\left(X, m\left(K_{X}+S+L^{\prime}\right)-k S\right) \rightarrow \bigoplus_{m, k} H^{0}\left(S, m\left(K_{S}+L^{\prime}\right)-k S_{\mid S}\right)\right)
$$

where $m \in d Z$ and $k$ varies independently. We observe that the bundles we are interested in can be written as

$$
m\left(K_{X}+S+\sum_{j=1}^{K} \mu^{j} Y_{j}^{\prime}+A-\frac{k}{m} S\right)
$$

and let $C_{A}$ be a large and divisible enough integer, such that $C_{A} A$ and $C_{A} A-S$ are very ample. Then we have

$$
\begin{aligned}
A-\frac{k}{m} S & =\frac{k}{m}\left(C_{A} A-S\right)+\left(\frac{1}{2}-\frac{k}{m} C_{A}\right) A+1 / 2 A \equiv \\
& \equiv \frac{k}{m} H_{1}+\left(\frac{1}{2 C_{A}}-\frac{k}{m}\right) H_{2}+1 / 2 A
\end{aligned}
$$

where $H_{1} \equiv C_{A} A-S$ and $H_{1} \equiv C_{A} A$ are hyperplane sections. Therefore, the bundle (2) becomes

$$
m\left(K_{X}+S+\sum_{j=1}^{K} \mu^{j} Y_{j}^{\prime}+l^{1}(\tau) H_{1}+l^{2}(\tau) H_{2}+A_{1}\right)
$$

(with the obvious notations from the relations above); the restricted algebra associated to it is identical to the one considered in 0.1 , modulo the fact that we have to assume that the ration $\tau=\frac{k}{m}$ is not too large (in order to insure the positivity of the quantities $l^{j}(\tau)$ ), and -which is more serious- the fact that we have to take into account the analogue of the divisor $\Delta_{S}$ in this new setting.

Indeed, we have $\Delta_{S}=\Delta_{S}(\tau)$, so we have to analyse the variation of the coefficients $\rho^{j}(\tau)$ of this divisor as functions of $\tau$. It turns out that the functions $\rho^{j}$ are piecewise affine, (as they should be, in view of the results of L. Ein et al., see [11]). At this point we follow closely the ideas in [19] which we describe now. For each $j$, the function $\rho^{j}(\tau)$ is obviously convex ; the important remark in [19] is that under 
some very precise hypothesis we obtain an inequality opposed to the one we get from convexity, as a consequence of the results concerning the extension of pluricanonical forms (the principle is absolutely the same as the one used in [14] for the rationality of the restricted algebras). It is for the proof of this affirmation that the polyhedral structure of the sets $\mathcal{E}_{Y, A}$ above is needed, in combination with the extension theorems for pluricanonical forms. Once the "affinity" statement is proved, it follows by induction that $\mathcal{R}^{\prime}$ is finitely generated, modulo sections whose normalized vanishing order along $S$ is larger than some positive constant.

Very roughly, we show next that it is possible to iterate this procedure, till we achieve finite generation of our initial algebra $\mathcal{R}$ modulo sections whose normalized vanishing order along each hypersurface $S_{1}, \ldots, S_{K}$, is greater than a fixed constant, where the $\left(S_{i}\right)$ are the support of the zero set of some fixed pluricanonical section. Then we are done.

Concerning the point i) in 0.1 , the approach is very clear : given that $\mathcal{E}_{Y, A}$ is a compact convex set, it is enough to show that its extremal points are isolated near any fixed $t_{0} \in \mathcal{E}_{Y, A}$. The important techniques in the proof of this affirmation are closely related to the pseudoeffectivity results which are implicit in [22] ; again, our presentation borrows some ideas from [19], even if the language used and the actual proof are quite different. We mention here that via the minimal model program one equally obtains a decomposition of the set $\mathcal{E}_{Y, A}$, together with a beautiful geometric interpretation of it (see [2], [16], [24]).

In conclusion, the extension theorems for pluricanonical forms are crucial for all the main steps of the arguments presented in this article. The structure of our proof shows clearly that in order to obtain new non-vanishing and/or finite generation results (e.g. using as little as possible of the positivity of the $\mathbb{Q}$-bundle $A$ ), this is the technique to be further investigated and refined.

\section{$\S 1$ Basic definitions and notations}

In this paragraph we fix some notations and we collect a few results which will be needed in the proof of 0.1 .

Let $\{\alpha\} \in \operatorname{Psef}(X)$ be a pseudoeffective cohomology class of $(1,1)$ type, where $\alpha$ is a non-singular and closed differential form. The important notion of current with minimal singularities in the sense of pluripotential theory associated to the class $\{\alpha\}$ was introduced in [7]. The corresponding object will be denoted by $\Theta_{\min }$, and will be refered here as current with minimal singularities in the sense of Demailly. We recall next its definition ; for a more ample presentation of its properties, see [4], [5], [7], [8], $[9]$.

We consider the family of potentials

$$
\mathcal{P}:=\left\{f \in L^{1}(X): f \leq 0 \text { and } \alpha+\sqrt{-1} \partial \bar{\partial} f \geq 0\right\}
$$

where the above inequality is assumed to hold in the sense of currents on $X$. Then we define $f_{\min }$ to be the regularized upper envelope of the above family, and then

$$
\Theta_{\min }:=\alpha+\sqrt{-1} \partial \bar{\partial} f_{\min } .
$$


In the geometric context $\{\alpha\}=c_{1}(L)$, where $L \rightarrow X$ is a $\mathbb{Q}$-bundle, one can restrict the family of functions $\mathcal{P}$ above, and only take into account the potentials $f$ induced by holomorphic sections of $L$ and its multiples (suitably normalized). Let $f_{\min , \sigma}$ be the regularized upper envelope of this smaller family (see [26]) ; the corresponding current is denoted by $\Theta_{\min , \sigma}$ and we call it current with minimal singularities in the sense of Siu. The inequality

$$
f_{\min } \geq f_{\min , \sigma}
$$

is obvious from the definitions. If the bundle $L$ is big, then the above currents have equivalent singularities if and only if the algebra of sections defined by $L$ is of finite type, as remarked in [5]. The relationship between these two currents play an important rôle in the proof of 0.1 .

Let $W \subset X$ be an irreducible hypersurface ; following [5], [20], we denote by $\nu_{W}(\{\alpha\})$ the minimal multiplicity of the class $\{\alpha\}$ along $W$. We recall that the minimal multiplicity $\nu_{W}(\{\alpha\})$ is in general strictly smaller than the Lelong number of the current with minimal singularities of $\{\alpha\}$, cf [5].

Next, we have the notion of numerical dimension of a pseudoeffective class $\{\alpha\}$ generalizing the corresponding definition in the setting of the nef line bundles. In the present text we will denote this invariant by $\operatorname{nd}(\{\alpha\})$.

We will not reproduce the precise definition of the preceding invariants ; their relevance for our article is explained in the next statement.

1.1 Theorem[5], [20]. Let $X$ be a compact complex manifold, and let $\{\alpha\} \in \operatorname{Psef}(X)$ be a pseudoeffective class.

(a) The function $\{\alpha\} \rightarrow \nu_{W}(\{\alpha\})$ is convex and lower semi-continuous on the closed set $\operatorname{Psef}(X)$.

(b) We assume that $\operatorname{nd}(\{\alpha\})=0$; then there exists an unique closed positive current

$$
T=\sum_{i=1}^{N} a_{T}^{j}\left[W_{j}\right] \in\{\alpha\}
$$

and moreover we have $a_{T}^{j}=\nu_{W_{j}}(\{\alpha\})$.

We recall next the notion of asymptotic vanishing order of a divisor along an irreducible hypersurface $W \subset X$.

Let $D$ be an effective $\mathbb{Q}$-divisor ; following [11], we define

$$
\operatorname{ord}_{W}(\|D\|):=\lim _{p \rightarrow \infty} \frac{\operatorname{ord}_{W}(|p D|)}{p}
$$

where $\operatorname{ord}_{W}(|p D|)$ is the vanishing order along $W$ of a generic representative of the linear system $|p D|$. The order function can be extended by continuity to the cone of effective $\mathbb{R}$-divisors ; we refer to [11] for the proof of this assertion.

The following important result was equally established in [11], section 4 . Let $D_{1}, \ldots, D_{r}$ be effective $\mathbb{Q}$-divisors, such that the Cox ring

$$
\mathcal{R}:=\bigoplus_{\left(m^{1} \ldots, m^{r}\right) \in d \mathbb{Z}_{+}} H^{0}\left(X, m^{1} D_{1}+\ldots+m^{r} D_{r}\right)
$$


is finitely generated, where $d$ is a divisible enough integer.

1.2 Theorem[11]. Under the above assumption, there exists a smooth fan $\Xi$ refining $\mathbb{N}^{r}$ such that on each cone of $\Xi$, the function

$$
\left(m^{1}, \ldots m^{r}\right) \rightarrow \operatorname{ord}_{W}\left(\left\|m^{1} D_{1}+\ldots+m^{r} D_{r}\right\|\right)
$$

is linear.

In particular, there exists a refinement $\left(\Delta_{j}\right)$ of the standard polytope

$$
\Delta:=\left\{\tau=\left(\tau^{1}, \ldots, \tau^{r}\right): \tau^{j} \geq 0, j=1, \ldots, r \text { and } \tau^{1}+\ldots+\tau^{r}=1\right\}
$$

such that each $\Delta_{j}$ is generated by finite set of vertices with rational coordinates, and such that if we denote by $D_{\tau}:=\sum \tau^{j} D_{j}$, then we have

$$
\operatorname{ord}_{W}\left(\left\|(1-\eta) D_{\tau_{0}}+\eta D_{\tau_{1}}\right\|\right)=(1-\eta) \operatorname{ord}_{W}\left(\left\|D_{\tau_{0}}\right\|\right)+\eta \operatorname{ord}_{W}\left(\left\|D_{\tau_{1}}\right\|\right)
$$

for any $\tau_{0}, \tau_{1}$ within the same $\Delta_{j}$, and any $0 \leq \eta \leq 1$. The equality (3) follows directly from the theorem 1.2 if $\tau_{1}, \tau_{2}$ and $\eta$ have rational coordinates ; in general, we use a continuity argument.

We note that if $D_{1}$ and $D_{2}$ are numerically equivalent big divisors, then we have $\operatorname{ord}_{W}\left(\left\|D_{1}\right\|\right)=\operatorname{ord}_{W}\left(\left\|D_{2}\right\|\right)$, as stated in the theorem A of [11]. If $D_{1}=K_{X}+L_{1}$ where $L_{1}$ is klt and big, then the same equality holds true, as soon as $D_{1}$ is (pseudo)effective (i.e. in this case we do not need the "big" hypothesis), see [22].

To conclude this paragraph, we collect next a few very elementary results concerning the set $\mathcal{E}_{Y, A}$ defined in the introduction by using the hypersurfaces $\left(Y_{j}\right)_{j=1, \ldots, N}$.

In order to study its "shape" near an arbitrary point $\tau_{0}$, it would be convenient to have $0 \leq \tau_{0}^{j} \leq 1-\varepsilon_{0}$, for some strictly positive $\varepsilon_{0}$ and all the indexes $j$. We show that we can assume that such inequalities holds, provided that we modify slightly the ample part $A$.

We use the following translation technique which goes back at least to [2], as follows. Let $0<\varepsilon_{0}<1 / 2$ be a rational number, such that $A+\varepsilon_{0} \sum_{j \in J} Y_{j}$ is still ample, for any subset $J \subset\{1, \ldots, N\}$. We define the set

$$
J_{0}:=\left\{1 \leq j \leq N: \tau_{0}^{j}>1-\varepsilon_{0}\right\}
$$

and then for any $\tau \in[0,1]^{N}$ we have

$$
K_{X}+\sum_{j \in\{1, \ldots, N\} \backslash J_{0}} \tau^{j} Y_{j}+\sum_{j \in J_{0}} \tau^{j} Y_{j}+A \equiv K_{X}+\sum_{j \in\{1, \ldots, N\} \backslash J_{0}} \tau^{j} Y_{j}+\sum_{j \in J_{0}}\left(\tau^{j}-\varepsilon_{0}\right) Y_{j}+A_{0}
$$

where $A_{0}$ is the ample bundle $A+\varepsilon_{0} \sum_{j \in J_{0}} Y_{j}$. We remark that $0 \leq \tau_{0}^{j}-\varepsilon_{0} \leq 1-\varepsilon_{0}$ for any $j \in J_{0}$, and therefore there exists a small open set $\Omega \subset \mathcal{E}_{Y, A}$ containing the point $\tau_{0}$, such that the map $\Phi: \Omega \rightarrow \mathcal{E}_{Y, A_{0}}$ given by the relation

$$
\tau \rightarrow\left(\tau^{\prime}, \tau^{\prime \prime}-\varepsilon_{0}\right)
$$


is well defined, where $\tau^{\prime}$ (respectively $\tau^{\prime \prime}$ ) corresponds to the components of $\tau$ whose indexes belongs to $\{1, \ldots, N\} \backslash J_{0}$ (respectively $J_{0}$ ).

In conclusion, while analysing the set $\mathcal{E}_{Y, A}$ near one of its points $\tau_{0}$, we can assume that $0 \leq \tau_{0}^{j} \leq 1-\varepsilon_{0}$, for all $1 \leq j \leq N$.

Let $\mu: \widehat{X} \rightarrow X$ be a composition of blow-up maps with non-singular centers, such that the proper transform of the divisor $\sum_{j=1}^{N} Y_{j}$ together with the support of the relative canonical bundle $K_{\widehat{X} / X}$ of $\mu$ have strictly normal crossings ; we denote by $\left(\widehat{Y}_{j}\right)_{j \in \widehat{J}}$ the corresponding hypersurfaces. We have

$$
\mu^{\star}\left(Y_{\tau}\right)=\sum_{j \in \widehat{J}} r^{j}(\tau) \widehat{Y}_{j}
$$

where the $r^{j}$ above are linear functions with rational and positive coefficients, completely determined by the map $\mu$ and the set $\left(Y_{j}\right)$. Next, we have

$$
\mu^{\star}\left(K_{X}+Y_{\tau}+A\right)=K_{\widehat{X}}+\sum_{j \in \widehat{J}}\left(r^{j}(\tau)-a_{\widehat{X} / X}^{j}\right) \widehat{Y}_{j}+\mu^{\star}(A)
$$

where the $\left(a_{\widehat{X} / X}^{j}\right)$ are the coefficients of the relative canonical divisor.

Since we can assume that $0 \leq \tau^{j} \leq 1-\varepsilon_{0} / 2$ if $\tau \in \Omega$, we have (see [17])

$$
r^{j}(\tau)-a_{\widehat{X} / X}^{j}<1
$$

for all $j \in \widehat{J}$; the inequalities above will still hold if we add any small enough $\delta^{j}$ to their left hand side. As it is well known, for any $\varepsilon>0$ there exists $0<\delta^{j}<\varepsilon$ such that

$$
\widehat{A}:=\mu^{\star}(A)-\sum_{j \in \widehat{J}} \delta^{j} \widehat{Y}_{j}
$$

is ample, and therefore we get

$$
\mu^{\star}\left(K_{X}+Y_{\tau}+A\right)=K_{\widehat{X}}+\sum_{j \in \widehat{J}}\left(r^{j}(\tau)-a_{\widehat{X} / X}^{j}+\delta^{j}\right) \widehat{Y}_{j}+\widehat{A}
$$

with the coefficients of $\widehat{Y}_{j}$ above strictly smaller than 1 .

In addition, by shrinking eventually the open set $\Omega$ near $\tau_{0}$, we can assume that $\widehat{J}$ is a disjoint union $\widehat{J}_{p} \cup \widehat{J}_{n}$ where $r^{j}(\tau)-a_{\widehat{X} / X}^{j}+\delta^{j}>0$ if and only if $j \in \widehat{J}_{p}$, for any $\tau \in \Omega$. We observe that the hypersurfaces $\widehat{Y}_{j}$ corresponding to $j \in \widehat{J}_{n}$ are $\mu$-exceptional ; the relation (7) becomes

(8) $\mu^{\star}\left(K_{X}+Y_{\tau}+A\right)+\sum_{j \in \widehat{J}_{n}}\left(a_{\widehat{X} / X}^{j}-\delta^{j}-r^{j}(\tau)\right) \widehat{Y}_{j}=K_{\widehat{X}}+\sum_{j \in \widehat{J}_{p}}\left(r^{j}(\tau)-a_{\widehat{X} / X}^{j}+\delta^{j}\right) \widehat{Y}_{j}+\widehat{A}$ 
By using the relation (8) above, we can define a map $\Phi_{1}: \Omega \rightarrow \mathcal{E}_{\widehat{Y}}, \widehat{A}$ as follows

$$
\tau \rightarrow\left(r^{j}(\tau)-a_{\widehat{X} / X}^{j}+\delta^{j}\right)_{j \in \widehat{J}_{p}}
$$

It is easy to see that $\Phi_{1}$ is well defined, and one can even describe its image thanks to the Hartogs principle.

Since $\Phi_{1}$ is an affine map defined over the rational numbers, the inverse image of any rational polytope is a rational polytope as well. We will make an extensive use of slightly more general considerations in a moment, but we have chosen to include this simple discussion here, as an illustration for what is about to follow.

\section{$\S 2$ Proof of (i)}

We start with a few preliminary remarks ; in the first place, the set $\mathcal{E}_{Y, A}$ is convex ; it is equally closed by the usual properties of the pseudoeffective cone.

Also, we note that a point $\tau_{0} \in \mathcal{E}_{Y, A}$ having at least one non-rational coordinate cannot be extremal. The argument is an immediate consequence of the non-vanishing theorem (see [2], [22]), as follows.

Let $\sum_{j \in I} \rho_{0}^{j}\left[W_{j}\right]$ be an effective $\mathbb{R}$-divisor, numerically equivalent with $K_{X}+Y_{\tau_{0}}+A$.

As in [22], paragraph 1.I, we consider the set

$$
\mathcal{J}:=\left\{(x, \tau) \in \mathbb{R}^{|I|} \times \mathbb{R}^{N}: \sum_{j \in I} x^{j}\left[W_{j}\right] \equiv K_{X}+Y_{\tau}+A\right\}
$$

and we note that $\mathcal{J}$ is an affine subspace of $\mathbb{R}^{|I|} \times \mathbb{R}^{N}$, which is defined over the rational numbers. Our given data $\left(\rho_{0}, \tau_{0}\right)$ corresponds to a point in $\mathcal{J}$; let $\eta>0$ be a positive number. We can construct the rational approximations $\left(\rho_{\eta s}, \tau_{\eta s}\right) \in \mathcal{J}$ of $\left(\rho_{0}, \tau_{0}\right)$ such that :

(i) There exists $q_{\eta s} \in \mathbb{Z}_{+}$such that $q_{\eta s}\left(\rho_{\eta s}, \tau_{\eta s}\right)$ has integer coordinates, and such that the next Dirichlet inequality is satisfied $q_{\eta s}\left\|\rho_{0}-\rho_{\eta s}\right\|<\eta$ (and a similar relation for the $\left.\tau_{0}\right)$.

(ii) The point $\left(\rho_{0}, \tau_{0}\right)$ belongs to the convex hull of $\left(\rho_{\eta s}, \tau_{\eta s}\right)$.

We remark that the coordinates of $\rho_{\eta s}$ are positive rational numbers, and we clearly have $\tau_{\eta s} \in([0,1] \cap \mathbb{Q})^{N}$ as soon as $\eta \ll 1$; this is a consequence of (i) above. In conclusion, if at least one component of $\tau_{0}$ is not rational, then this point cannot be extremal.

By the classical theory of convex sets, the first part of 0.1 can be reformulated as follows.

2.1 Claim. The set of extremal points of the set $\mathcal{E}_{Y, A}$ is isolated.

Our proof of the claim 2.1 is in some sense a generalization of the arguments in [22] : there are two main cases we are forced to consider. 
First we analyse an extremal point $\tau_{0} \in \mathcal{E}_{Y, A}$ for which the numerical dimension of the corresponding $\mathbb{Q}$-bundle $K_{X}+Y_{\tau_{0}}+A$ is equal to zero. In order to establish the claim under this hypothesis, the main results we invoke here are due to S. Boucksom (see [5]).

If the numerical dimension of the bundle $K_{X}+Y_{\tau_{0}}+A$ is at least one, then given any sequence $\tau_{k} \rightarrow \tau_{0}$ as $k \rightarrow \infty$ we will create a center $S$ adapted to $\left(\tau_{k}\right)$ on a modification of $X$, and we will use the full force of 0.1 in lower dimensions for the proof of 2.1 .

Remark. In the article [19], the author follows a different path for the proof of the statement 2.1. One of the important steps in his approach is that the limit of any nonconstant sequence of extremal points is necessarily nef in codimension 2. Unfortunately we do not understand the arguments he invoke to prove this fact in at the page 35 in [19], proof of the step 3 (but of course, the fact itself is true a posteriori). Nevertheless, some ideas/techniques from [19] are crucial for the proof of the statements 2.C.6 and 2.C.7.

\section{§2.A The case nd $\left(\left\{K_{X}+Y_{\tau_{0}}+A\right\}\right)=0$}

Let $\tau_{0} \in \mathcal{E}_{Y, A}$ be a point such that nd $\left(\left\{K_{X}+Y_{\tau_{0}}+A\right\}\right)=0$; we do not assume it rational or extremal, for the moment. By the result 1.1 recalled in the preceding paragraph, there exists an unique closed positive current

$$
\Theta_{0}:=\sum_{j \in I} a_{\min }^{j}\left(\tau_{0}\right)\left[W_{j}\right]
$$

in the class $K_{X}+Y_{\tau_{0}}+A$; we denote by $I$ above a finite set.

Let $\tau_{k} \in \mathcal{E}_{Y, A}$ be a sequence converging to $\tau_{0}$ as $k \rightarrow \infty$, and let

$$
\Theta_{k}:=\sum_{j \in I} a_{\min }^{j}\left(\tau_{k}\right)\left[W_{j}\right]+\Lambda_{k}
$$

be a current with minimal singularities in the class $\left\{K_{X}+Y_{\tau_{k}}+A\right\}$; along the next few lines, we reproduce the arguments in [5] to show that we have $\lim _{k} a_{\min }^{j}\left(\tau_{k}\right)=a_{\min }^{j}\left(\tau_{0}\right)$.

In the first place, the sequence of currents $\Theta_{k}$ (can be assumed to) converge to $\Theta_{0}$. Indeed this is clear, by the uniqueness part of the statement 1.1 above, combined with standard properties of closed positive currents, see [25]. By the semi-continuity of the Lelong numbers of closed positive currents, we have

$$
a_{\min }^{j}\left(\tau_{0}\right) \geq \lim _{k} a_{\min }^{j}\left(\tau_{k}\right)
$$

On the other hand, for each $k \geq 1$ and each index $j$ we have

$$
a_{\min }^{j}\left(\tau_{k}\right) \geq \nu_{W_{j}}\left(\left\{K_{X}+Y_{\tau_{k}}+A\right\}\right) .
$$

This inequality combined with the lower-semicontinuity result 1.1 (a) yields

$$
a_{\min }^{j}\left(\tau_{0}\right) \leq \lim _{k} a_{\min }^{j}\left(\tau_{k}\right)
$$


and thus we obtain

$$
a_{\min }^{j}\left(\tau_{0}\right)=\lim _{k} a_{\min }^{j}\left(\tau_{k}\right)
$$

For any positive real $\eta>0$, we consider the current

$$
T_{k, \eta}:=\Theta_{k}+\eta\left(\Theta_{k}-\Theta_{0}\right)
$$

Then we have $T_{k, \eta} \in\left\{K_{X}+Y_{\tau_{k, \eta}}+A\right\}$, where $\tau_{k, \eta}:=\tau_{k}+\eta\left(\tau_{k}-\tau_{0}\right)$. The next simple considerations enable us to conclude.

- The components of $\tau_{k, \eta}$ belong to the interval [0,1], as soon as $k \gg 0$ (we recall that the components of $\tau_{0}$ are assumed to be positive and smaller than $1-\varepsilon_{0}$ ).

- The current $T_{k, \eta}$ is positive-it is at this point that we are using the equality (12) in an essential manner.

In conclusion, the quantity $\tau_{k}$ can be written as a convex combination of $\tau_{0}$ and $\tau_{k, \eta}$; each of these two points belong to $\mathcal{E}_{Y, A}$ and neither of them is equal to $\tau_{k}$. Therefore the set of extremal points of $\mathcal{E}_{Y, A}$ is isolated at any $\tau_{0}$ for which the associated class $\left\{K_{X}+Y_{\tau_{0}}+A\right\}$ has numerical dimension zero.

\section{$\S 2 . B$ The " $x$ method" for sequences}

During the paragraphs 2.B and 2.C, our main goal will be to analyse the set of extremal points near $\tau_{0} \in \mathcal{E}_{Y, A}$, such that

$$
\operatorname{nd}\left(\left\{K_{X}+Y_{\tau_{0}}+A\right\}\right) \geq 1 \text {. }
$$

Nevertheless, we will try to develop a general setting, which will be useful for the proof of the second part of 0.1 .

Let $\tau_{k} \in \mathcal{E}_{Y, A}$ be a sequence of points converging to $\tau_{0}$ as $k \rightarrow \infty$; for the moment, we do not assume that $\left(\tau_{k}\right)$ are rational or extremal. In this subsection we will show that we can apply the so-called $x$-method in the version explained in [2] and [22], simultaneously for (a subsequence of) $\left(K_{X}+Y_{\tau_{k}}+A\right)_{k \in \mathbb{N}}$. There are practically no additional difficulties while transposing the arguments from the case of a single point to our set-up, but unfortunately there are many things to be verified, and the presentation is quite messy...

We can assume that the coordinates of the vector $\tau_{0} \in \mathcal{E}_{Y, A}$ belong to the interval $\left[0,1-\varepsilon_{0}\right]$, for some $\varepsilon_{0}>0$, thanks to the discussion at the end of the paragraph 1 . Along the next few lines, we construct the analogue of an incomplete linear system in the usual x-method corresponding to each $\tau_{k}$, whose singularities do not change as $k \rightarrow \infty$.

The class $\left\{K_{X}+Y_{\tau_{k}}+A\right\}$ is pseudoeffective, and we denote by $\Theta_{k}$ its current with minimal singularities in the sense of Demailly. There exists $x_{0} \in X$ a very general point, such that $\nu\left(\Theta_{k}, x_{0}\right)=0$, for any $k \geq 0$. By the folklore results recalled e.g. in 
[18], there exists a Kähler current $T_{0} \in\left\{m_{0}\left(K_{X}+Y_{\tau_{0}}+A\right)+A\right\}$, with logarithmic poles and such that

$$
\nu\left(T_{0}, x_{0}\right) \geq \operatorname{dim}(X)+1
$$

where $m_{0}$ is a large enough integer. We can also assume that the singularities of $T_{0}$ are rational numbers, in the sense that there exists a birational map $f: \widehat{X} \rightarrow X$ such that $f^{\star}\left(T_{0}\right)$ can be written as an effective divisor with rational coefficients plus a non-singular $(1,1)$-form. We define

$$
T_{k}:=T_{0}+\sum_{j=1}^{N}\left(\tau_{k}^{j}-\tau_{0}^{j}\right) \theta_{j},
$$

where $\theta_{j}$ is a non-singular representative of $m_{0} c_{1}\left(Y_{j}\right)$. If the components of the vector $\tau_{0}$ happen to be rational numbers, then the current $T_{k}$ defined above is convenient for our future purposes. In general, we have to modify it further, as follows.

We denote by $\omega_{A} \in c_{1}(A)$ a Kähler form ; let $C_{0} \in \mathbb{N}$ be a large enough integer, such that $m_{0} Y_{j}+C_{0} A$ and $C_{0} A$ are very ample. We consider $H_{j}$, respectively $H_{0}$ generic hyperplane sections of these bundles.

For each $\eta>0$, we consider a vector $\tau_{\eta} \in[0,1]^{N}$, with rational coordinates, such that $\left\|\tau_{\eta}-\tau_{0}\right\| \leq \eta$. We define the affine forms $f^{j}(\tau)=\tau^{j}-\tau_{\eta}^{j}+2 \eta$, where $j=1, \ldots, N$, and respectively $f^{0}(\tau)=\sum_{n=1}^{N}\left(2 \eta+\tau_{\eta}^{n}-\tau^{n}\right)$ and then we have

$$
\begin{aligned}
T_{k} & =T_{0}+\sum_{j=1}^{N}\left(\tau_{\eta}^{j}-\tau_{0}^{j}\right) \theta_{j}+\sum_{j=1}^{N}\left(\tau_{k}^{j}-\tau_{\eta}^{j}\right) \theta_{j}= \\
& =T_{\eta}+\sum_{j=1}^{N}\left(\tau_{k}^{j}-\tau_{\eta}^{j}+2 \eta\right) \theta_{j}-2 \eta \sum_{j=1}^{N} \theta_{j} \equiv \\
& \equiv T_{\eta}-2 \eta\left(2 C_{0} N \omega_{A}+\sum_{j=1}^{N} \theta_{j}\right)+\sum_{j=1}^{N} f^{j}\left(\tau_{k}\right) H_{j}+f^{0}\left(\tau_{k}\right) H_{0}
\end{aligned}
$$

In the above equalities, we denote by $T_{\eta}:=T_{0}+\sum_{j=1}^{N}\left(\tau_{\eta}^{j}-\tau_{0}^{j}\right) \theta_{j}$; we see that we have $0 \leq f^{j}\left(\tau_{k}\right) \leq 4 \eta$, if $k \gg 0$.

We remark that there exists a positive $\delta_{0}$ such that $T_{\eta} \geq \delta_{0} \omega_{A}$ for any $\eta$ small enough. In conclusion, we can choose $\eta:=\eta_{0}$ a positive rational number, such that

$$
\Xi:=T_{\eta_{0}}-2 \eta_{0}\left(2 C_{0} N \omega_{A}+\sum_{j=1}^{N} \theta_{j}\right)
$$

is positive, and its we observe that its cohomology class is rational. The current $\Xi$ has the same singularities as $T_{0}$, and we re-define $T_{k}$ as follows

$$
T_{k}:=\Xi+\sum_{j=0}^{N} f^{j}\left(\tau_{k}\right)\left[H_{j}\right] ;
$$


it belongs to the class $m_{0}\left(K_{X}+Y_{\tau_{k}}+A\right)+A$.

We consider a log-resolution $\widetilde{\mu}: \widetilde{X} \rightarrow X$ of $T_{0}+\sum_{j=1}^{N} Y_{j}$. The $\widetilde{\mu}$-inverse image of the objects which will come next into the picture can be written as follows.

$$
\tilde{\mu}^{\star}\left(T_{k}\right)=\sum_{i \in I} a_{T}^{i}\left[\widetilde{Y}_{i}\right]+\Lambda_{\Xi}+\sum_{j=0}^{N} f^{j}\left(\tau_{k}\right)\left[\bar{H}_{j}\right]
$$

where $\Lambda_{\Xi}$ is a semi-positive form whose cohomology class is rational, the coefficients $a_{T}^{i}$ are positives and rational, and $\bar{H}_{j}$ is the proper transform of $H_{j}$, which can be assumed to be equal to the total transform of $H_{j}$, since these hypersurfaces are generic.

Similarly, we write

$$
\widetilde{\mu}^{\star}\left(Y_{\tau_{k}}\right)=\sum_{i \in I} l^{i}\left(\tau_{k}\right) \tilde{Y}_{i}
$$

where $l^{i}: \mathbb{R}^{N} \rightarrow \mathbb{R}$ are affine functions with positive and rational coefficients. The relative canonical bundle of $\widetilde{\mu}$ is written as follows

$$
K_{\widetilde{X} / X}=\sum_{i \in I} a_{\widetilde{X} / X}^{i}\left[\widetilde{Y}_{i}\right]
$$

As in the previous section, we denote by $\Theta_{k}$ a current with minimal singularities in the sense of Demailly of the class $\left\{K_{X}+Y_{\tau_{k}}+A\right\}$; its $\widetilde{\mu}$-inverse image can be decomposed according to the set of $\left(\widetilde{Y}_{i}\right)$, as follows :

$$
\tilde{\mu}^{\star}\left(\Theta_{k}\right)=\sum_{i \in I} a_{\min }^{i}\left(\tau_{k}\right)\left[\tilde{Y}_{i}\right]+\Lambda_{k}
$$

where $a_{\min }^{i}\left(\tau_{k}\right)$ are positive real numbers, and where $\Lambda_{k}$ is a closed positive current, whose generic Lelong number along each of $\left(\widetilde{Y}_{i}\right)$ is equal to zero.

A slight difficulty in what will follow is the fact that a-priori, we ignore the variation of the quantity $a_{\min }^{i}\left(\tau_{k}\right)$ with respect to $k$, but still it is a bounded sequence, and for our purposes we can assume that $a_{\text {min }}^{i}\left(\tau_{k}\right) \rightarrow a_{\infty}^{i}$ as $k \rightarrow \infty$. We remark that at this point there is no connection between $a_{\infty}^{i}$ and the expected singularity $a_{\min }^{i}\left(\tau_{0}\right)$ of the minimal current of the class $\left\{K_{X}+Y_{\tau_{0}}+A\right\}$.

We have the next statement, which is a first step towards the proof of the claim 2.1.

2.B.1 Lemma. Under the assumptions and notations above, there exists $t^{0}, q^{j} \in \mathbb{Q}_{+}$, a family of affine forms $r^{i}: \mathbb{R}^{1+N} \rightarrow \mathbb{R}$ with rational coefficients, and a set of strictly normal crossings hypersurfaces $\left(\widetilde{Y_{j}}\right)_{j \in \widetilde{I}} \subset \widetilde{X}$ such that

$$
\left(1+m_{0} t^{0}\right) \Lambda_{k}+\sum_{i \in I_{n} \cup I_{p}} \widetilde{l}_{k}^{i}\left(\tau_{k}\right)\left[\widetilde{Y}_{i}\right] \equiv K_{\widetilde{X}}+\widetilde{S}+\sum_{i \in I_{p}} r^{i}\left(\tau_{k}, a_{\min }^{0}\left(\tau_{k}\right)\right) \widetilde{Y}_{i}+\widetilde{A}_{1}
$$


where $\widetilde{I}=I_{p} \cup I_{n} \cup\{0\}$ is a partition of $\widetilde{I}$, and where we use the next notations

$$
\widetilde{l_{k}^{i}}(\tau):=\left(1+m_{0} t^{0}\right) a_{\min }^{j}\left(\tau_{k}\right)+a_{\widetilde{X} / X}^{j}-t^{0} a_{\Xi}^{j}-l^{j}(\tau)
$$

for $i \in I_{n}$, as well as

$$
\widetilde{l_{k}^{i}}(\tau):=\left(1+m_{0} t^{0}\right)\left(a_{\min }^{j}\left(\tau_{k}\right)-q^{j}\right)
$$

for $i \in I_{p}$; we also denote by $\widetilde{S}:=\widetilde{Y_{0}}$. In addition, the following relations holds true provided that $k$ belongs to a well chosen subsequence of natural numbers.

(a) For each $j \in I_{n} \cup I_{p}$ we have $0 \leq \widetilde{l_{k}^{i}}\left(\tau_{k}\right) \leq\left(1+m_{0} t^{0}\right) a_{\min }^{j}\left(\tau_{k}\right)+a_{\widetilde{X} / X}^{j}$.

(b) For each $i \in I_{p}$ we have $0 \leq r^{i}\left(\tau_{k}, a_{\min }^{i}\left(\tau_{k}\right)\right) \leq 1-\varepsilon_{0}$, where $\varepsilon_{0}$ is a positive real number.

(c) The bundle $\widetilde{A}$ is ample on $\widetilde{X}$.

The approach we will follow for the proof is quite clear : first we determine the center $\widetilde{S}$ by using the limit bundle $K_{X}+Y_{\tau_{0}}+A$, and then thanks to the fact that the family of "incomplete linear systems" $T_{k}$ we have just constructed is explicit enough, we can describe in a very precise manner the variation of the coefficients when we replace $\tau_{0}$ by $\tau_{k}$.

Proof. To start with, we remark that for any real number $t$ we have the next numerical identity (see [22] for similar considerations) :

$$
\begin{aligned}
\left(1+m_{0} t\right) \Lambda_{k} & \equiv K_{\widetilde{X}}+\sum_{i \in I}\left(t a_{\Xi}^{i}+l^{i}\left(\tau_{k}\right)-\left(1+m_{0} t\right) a_{\min }^{i}\left(\tau_{k}\right)-a_{\widetilde{X} / X}^{i}\right) \widetilde{Y}_{i}+ \\
& +t\left(\Lambda_{\Xi}+\sum_{j=0}^{N} f^{j}\left(\tau_{k}\right)\left[\bar{H}_{j}\right]\right)+(1-t) \mu^{\star}(A)
\end{aligned}
$$

Next, we will modify the expression (23) above and replace $\mu^{\star}(A)$ by

$$
\widetilde{A}:=\mu^{\star}(A)-\sum_{i \in I} \delta^{i} \widetilde{Y}_{i}
$$

(where $\delta^{i}>0$ and small enough). We still denote by $l^{i}$ the affine form obtained from $l^{i}$ by adding the constant $\delta^{i}$, and by $a_{\Xi}^{i}$ the real number $a_{\Xi}^{i}-\delta^{i}$, so that the cohomology class is unchanged after the above substitution.

We note that if the perturbation $\left(\delta^{i}\right)$ is small enough, then the coefficients of $\tilde{Y}_{i}$ above will still be strictly smaller than 1 for $t=0$, whereas for $t=1$, at least one of these numbers is strictly greater than 1 (given the singularities of $\Xi$ ). Furthermore, it is possible to choose the parameters $\left(\delta^{i}\right)$ such that the next quantities are distinct

$$
\widetilde{t}_{i}:=\frac{1+a_{\infty}^{i}+a_{\widetilde{X} / X}^{i}-l^{i}\left(\tau_{0}\right)}{a_{\Xi}^{i}-m_{0} a_{\infty}^{i}}
$$


for all $i \in I$.

We consider the set $I^{\prime}:=\left\{i \in I: a_{\Xi}^{i}-m_{0} a_{\infty}^{i}<0\right\}$; we cannot have $I^{\prime}=I$ by the choice of the point $x_{0}$. For all $k \gg 0$ we will have

$$
a_{\Xi}^{i}-m_{0} a_{\min }^{i}\left(\tau_{k}\right)<0
$$

if $i \in I^{\prime}$, as well as

$$
a_{\Xi}^{i}-m_{0} a_{\min }^{i}\left(\tau_{k}\right)>0
$$

provided that $i \in I \backslash I^{\prime}$.

The quantity $(24)_{i \in I \backslash I^{\prime}}$ will be minimal for an unique index $i=0$, and we denote by $\widetilde{S}$ the corresponding hypersurface. Then we have

$$
\widetilde{t}_{0} a_{\Xi}^{i}+l^{i}\left(\tau_{k}\right)-\left(1+m_{0} \widetilde{t}_{0}\right) a_{\min }^{i}\left(\tau_{k}\right)-a_{\widetilde{X} / X}^{i} \leq 1-\varepsilon_{0}
$$

for every $\underset{t_{0}}{j} \neq 0$, where $\varepsilon_{0}<1 / 4$ is a positive real number. By the above considerations, we have $\widetilde{t}_{0}<1$, but it may happen that this quantity is non-rational-and this will affect the rationality part of the lemma we want to obtain. In order to bypass this difficulty, we will consider an approximation of it, which is accurate enough for our further purposes.

For every rational number $\eta>0$, let $\widetilde{t}_{\eta} \in \mathbb{Q}$, such that $\widetilde{t}_{0}-\eta<\widetilde{t}_{\eta}<\widetilde{t}_{0}$. We consider the affine form

$$
\widetilde{f}(\tau, a):=1+\left(1+m_{0} \widetilde{t}_{\eta}\right) a+a_{\widetilde{X} / X}^{0}-\widetilde{t}_{\eta} a_{\Xi}^{0}-l^{0}(\tau) .
$$

A quick computation shows the existence of a constant $C_{0}>0$ such that

$$
-C_{0} \eta \leq \tilde{f}\left(\tau_{k}, a_{\min }^{0}\left(\tau_{k}\right)\right) \leq C_{0} \eta
$$

provided that $k \geq k_{\eta}$ is large enough.

By increasing $C_{0}$ if necessary, we assume that $C_{0} \widetilde{A}-\widetilde{Y}_{0}$ and $C_{0} \widetilde{A}$ are very ample ; we have

$$
\begin{aligned}
& \left(1-\widetilde{t}_{\eta}\right) \widetilde{A}-\widetilde{f}\left(\tau_{k}, a_{\min }^{0}\left(\tau_{k}\right)\right) \widetilde{Y}_{0}=\left(f\left(\tau_{k}, a_{\min }^{0}\left(\tau_{k}\right)\right)+C_{0} \eta\right)\left(C_{0} \widetilde{A}-\widetilde{Y}_{0}\right)+ \\
& \quad+\left(\frac{1}{2 C_{0}}\left(1-\widetilde{t}_{\eta}\right)-\widetilde{f}\left(\tau_{k}, a_{\min }^{0}\left(\tau_{k}\right)\right)\right) C_{0} \widetilde{A}+\frac{1}{2}\left(1-\widetilde{t}_{\eta}\right) \widetilde{A}-\eta C_{0}\left(C_{0} \widetilde{A}-Y_{0}\right) \\
& \quad \equiv f^{N+1}\left(\tau_{k}, a_{\min }^{0}\left(\tau_{k}\right)\right) \bar{H}_{N+1}+f^{N+2}\left(\tau_{k}, a_{\min }^{0}\left(\tau_{k}\right)\right) \bar{H}_{N+2}+ \\
& \quad+\frac{1}{2}\left(1-\widetilde{t}_{\eta}\right) \widetilde{A}-\eta C_{0}\left(C_{0} \widetilde{A}-Y_{0}\right)
\end{aligned}
$$

where $\bar{H}_{N+1} \equiv C_{0} \widetilde{A}-\widetilde{Y_{0}}$ and respectively $\bar{H}_{N+2} \equiv C_{0} \widetilde{A}$ are generic hyperplane sections, and the corresponding $f^{i}$ are affine forms with rational coefficients defined by the relation (29) above. 
We want the values of the affine forms $f^{i}$ to be positive and not too large when computed on $\left(\tau_{k}, a_{\text {min }}^{0}\left(\tau_{k}\right)\right)$, thus we define $\eta_{0}$ to be a positive rational number such that

$$
1-\widetilde{t}_{0} \geq 2 C_{0}^{2} \eta_{0}
$$

and such that $\frac{1}{2}\left(1-\widetilde{t}_{0}\right) \widetilde{A}+\widetilde{t}_{0} \Lambda_{\Xi}-C_{0} \eta_{0}\left(C_{0} \widetilde{A}-Y_{0}\right)$ is ample (this condition will be needed later). Let $t^{0}:=\widetilde{t}_{\eta_{0}}$ be any positive rational number in the interval $] \widetilde{t}_{0}-\eta_{0}, \widetilde{t}_{0}[$; with this choice, we note that we have

$$
0 \leq f^{j}\left(\tau_{k}, a_{\min }^{0}\left(\tau_{k}\right)\right) \leq 1-\widetilde{t}_{0}
$$

if $k \geq k_{\eta}$.

The next version of the inequality $(27)$ holds, since $t^{0} \leq \widetilde{t}_{0}$,

$$
t^{0} a_{\Xi}^{i}+l^{i}\left(\tau_{k}\right)-\left(1+m_{0} t^{0}\right) a_{\min }^{i}\left(\tau_{k}\right)-a_{\widetilde{X} / X}^{i} \leq 1-\varepsilon_{0} / 2
$$

for all $i \neq 0$ and for all $k \geq k_{\eta}$.

There are still a few cosmetic modifications we have to operate, in order to replace $a_{\min }^{i}\left(\tau_{k}\right)$ with a rational number independent of $k$.

Let $q^{j} \in \mathbb{Q}_{+}$be a rational number, such that for each $j \in I \backslash\{0\}$ and every $k$ large enough, we have

(a) $q^{j} \leq a_{\min }^{i}\left(\tau_{k}\right)$;

(b) $t^{0} a_{\Xi}^{i}+l^{i}\left(\tau_{k}\right)-\left(1+m_{0} t^{0}\right) q^{i}-a_{\widetilde{X} / X}^{i} \leq 1-\varepsilon_{0} / 4$.

We rewrite the relation (23) with $t=t^{0}$ in a modified form, according to (29).

$$
\begin{aligned}
& \left(1+m_{0} t^{0}\right)\left(\Lambda_{k}+\sum_{i \in I \backslash\{0\}}\left(a_{\min }^{i}\left(\tau_{k}\right)-q^{i}\right)\left[\widetilde{Y}_{i}\right]\right) \equiv K_{\widetilde{X}}+\widetilde{S}+ \\
& +\sum_{i \in I \backslash\{0\}}\left(t^{0} a_{\Xi}^{i}+l^{i}\left(\tau_{k}\right)-\left(1+m_{0} t^{0}\right) q^{i}-a_{\widetilde{X} / X}^{i}\right) \widetilde{Y}_{i}+\sum_{j=0}^{N+2} f^{i}\left(\tau_{k}, a_{\min }^{0}\left(\tau_{k}\right)\right) \overline{H_{i}}+\widetilde{A}_{1}
\end{aligned}
$$

where $\widetilde{A}_{1}: \equiv \frac{1}{2}\left(1-t^{0}\right) \widetilde{A}+t^{0} \Lambda_{\Xi}-C_{0} \eta_{0}\left(C_{0} \widetilde{A}-Y_{0}\right)$ is an ample $\mathbb{Q}$-bundle, and where we have replaced $t^{0} f^{j}$ by $f^{j}$ for notation's sake.

For $k \gg 0$, the coefficients

$$
t^{0} a_{\Xi}^{i}+l^{i}\left(\tau_{k}\right)-\left(1+m_{0} t^{0}\right) q^{i}-a_{\widetilde{X} / X}^{i}
$$

corresponding to $\widetilde{Y}_{i}$ above will be assumed to be negative if $i \in I_{+}$and positive if $i \in I_{-}$; moreover, we can assume that $I \backslash\{0\}=I_{+} \cup I_{-}$. Then the identity above becomes

$$
\left(1+m_{0} t^{0}\right) \Lambda_{k}+\sum_{i \in I_{n} \cup I_{p}} \widetilde{l_{k}^{i}}\left(\tau_{k}\right)\left[\widetilde{Y}_{i}\right] \equiv K_{\widetilde{X}}+\widetilde{S}+\sum_{i \in I_{p}} r^{i}\left(\tau_{k}, a_{\min }^{0}\left(\tau_{k}\right)\right) \widetilde{Y}_{i}+\widetilde{A}_{1}
$$


where for $i \in I_{+}$we introduce $r^{i}(\tau, a):=t^{0} a_{\Xi}^{i}+l^{i}(\tau)-\left(1+m_{0} t^{0}\right) q^{i}-a_{\widetilde{X} / X}^{i}$, and for $i=1, \ldots, N+3$, we have $r^{i}:=f^{i}$. We define $I_{n}:=I_{-}$, and $I_{p}:=I_{+} \cup\{1, \ldots, N+3\}$.

This is precisely the expression we seek ; the verification of the properties (a), (b) and (c) in the statement 2.B.1 is immediate, so we skip it.

Concerning the formula (33), we note the following interesting facts. Let $\tau \in[0,1]^{N}$ and $a \in \mathbb{R}_{+}$; we define the $\mathbb{R}$-divisor

$$
\begin{aligned}
E(\tau, a): & =\left(a_{\widetilde{X} / X}^{0}+\left(1+m_{0} t^{0}\right) a\right) \widetilde{S}+ \\
& +\sum_{i \in I_{n}}\left(t^{0} a_{\Xi}^{j}+l^{j}(\tau)\right) \widetilde{Y_{j}}+\sum_{i \in I_{p}}\left(\left(1+m_{0} t^{0}\right) q^{j}+a_{\widetilde{X} / X}^{j}\right) \widetilde{Y_{j}}
\end{aligned}
$$

As one can easily see, the support of the effective divisor $E(\tau, a)$ is not necessarily contractible with respect to $\widetilde{\mu}$. ¿From the formulas (19), (20) and (33) we infer the next numerical equivalence relation

$$
\begin{array}{r}
E\left(\tau_{k}, a_{\min }^{0}\left(\tau_{k}\right)\right)+K_{\widetilde{X}}+\widetilde{S}+\sum_{i \in I_{p}} r^{i}\left(\tau_{k}, a_{\min }^{0}\left(\tau_{k}\right)\right) \widetilde{Y}_{i}+\widetilde{A}_{1} \equiv \\
\equiv\left(1+m_{0} t^{0}\right) \mu^{\star}\left(K_{X}+Y_{\tau_{k}}+A\right)+K_{\widetilde{X} / X}
\end{array}
$$

and moreover we have the following correspondence between currents.

Let $T \in\left\{K_{X}+Y_{\tau_{k}}+A\right\}$ be a closed positive current ; then

$$
\widetilde{T} \in\left\{K_{\widetilde{X}}+\widetilde{S}+\sum_{i \in I_{p}} r^{i}\left(\tau_{k}, a_{\min }^{0}\left(\tau_{k}\right)\right) \widetilde{Y}_{i}+\widetilde{A}\right\}
$$

defined by the formula

$$
\left(1+m_{0} t^{0}\right) \widetilde{\mu}^{\star}(T)+\sum_{i \in I} a_{\widetilde{X} / X}^{i}\left[\widetilde{Y}_{i}\right]-E\left(\tau_{k}, a_{\min }^{0}\left(\tau_{k}\right)\right):=\widetilde{T}
$$

is positive, because

$$
E\left(\tau_{k}, a_{\min }^{0}\left(\tau_{k}\right)\right) \leq\left(1+m_{0} t^{0}\right) \tilde{\mu}^{\star}\left(\Theta_{k}\right)+\sum_{i \in I} a_{\widetilde{X} / X}^{i}\left[\tilde{Y}_{i}\right]
$$

in the sense of currents on $\widetilde{X}$. In particular, this shows that the current

$$
\widetilde{\Theta}_{k}:=\left(1+m_{0} t^{0}\right) \Lambda_{k}+\sum_{i \in I_{n} \cup I_{p}} \widetilde{l_{k}^{i}}\left(\tau_{k}\right)\left[\widetilde{Y}_{i}\right]
$$

is a current with minimal singularities of the class

$$
\left\{K_{\widetilde{X}}+\widetilde{S}+\sum_{i \in I_{p}} r^{i}\left(\tau_{k}, a_{\min }^{0}\left(\tau_{k}\right)\right) Y_{i}+\widetilde{A}\right\} .
$$


We recall now the following result : there exists a birational map $\widehat{\mu}: \widehat{X} \rightarrow \widetilde{X}$ such that the inverse image of $\widetilde{S}$ is equal to its proper transform, and such that for any set of real numbers $0 \leq x^{i} \leq 1-\varepsilon_{0}$ we have

$$
\widehat{\mu}^{\star}\left(K_{\widetilde{X}}+\widetilde{S}+\sum_{i \in I} x^{i} \widetilde{Y}_{i}\right)-\sum_{j \in J_{1}} \widehat{r}^{i}(x) E_{i} \equiv K_{\widehat{X}}+\widehat{S}+\sum_{j \in J_{2}} \widehat{r}^{i}(x) \widehat{Y}_{i} .
$$

where $E_{i}$ are $\widehat{\mu}$-exceptional, the $\widehat{Y}_{i}$ are mutually disjoint, and $\widehat{r}^{i}$ are affine forms, such that $\widehat{r}^{i}(x) \geq 0$ if and only if $i \in J_{2}$. We stress on the fact that in the formula above, only the decomposition $J_{1} \cup J_{2}$ depends on the particular vector $x$ we are dealing with, provided that $\varepsilon_{0}$ is fixed ; this is just a small variation on the very classical result stating the same thing without pointing out the (in)dependence of the data $\left(\widehat{\mu}, \widehat{r}^{i}\right)$ on the particular sequence $\left(x^{i}\right)$. Therefore we will just refer to the arguments provided in [14], from which the previous statement follows.

In our particular case, this translates as follows. There exists a map $\widehat{\mu}: \widehat{X} \rightarrow \widetilde{X}$, together with a set of non-singular and mutually disjoint hypersurfaces $\left(\widehat{Y}_{j}\right)$ such that

$$
\widehat{\mu}^{\star}\left(\widetilde{\Theta}_{k}\right)+\sum_{j \in J_{n}} \widehat{l}^{j}\left(\tau_{k}, a_{\min }^{0}\left(\tau_{k}\right)\left[E_{i}\right] \equiv K_{\widehat{X}}+S+\sum_{j \in J_{p}} \widehat{r}^{j}\left(\tau_{k}, a_{\min }^{0}\left(\tau_{k}\right) \widehat{Y}_{i}+\mu^{\star}(\widetilde{A}),\right.\right.
$$

where $\left(\widehat{r}^{j}, \widehat{l}^{j}\right)$ are affine forms defined over $\mathbb{Q}$, and $\left(E_{j}\right)$ are contracted by $\widehat{\mu}$; we list along the following lines the properties of the objects we have constructed so far, as an introduction for the next subsection.

B.1 For each $k$ large enough, we have

$$
1-\varepsilon_{0} \geq \widehat{r}^{i}\left(\tau_{k}, a_{\min }^{0}\left(\tau_{k}\right)\right) \geq 0 \text { and } \widehat{l}^{i}\left(\tau_{k}, a_{\min }^{0}\left(\tau_{k}\right)\right) \geq 0
$$

B.2 The class $\alpha\left(\tau_{k}, a_{\text {min }}^{0}\left(\tau_{k}\right)\right):=\left\{K_{\widehat{X}}+S+\sum_{j \in J_{p}} \widehat{r}^{i}\left(\tau_{k}, a_{\min }^{0}\left(\tau_{k}\right)\right) \widehat{Y}_{i}+\mu^{\star}(\widetilde{A})\right\}$ is pseudoeffective, and the Lelong number along $S$ of its minimal singularity current is equal to zero.

B.3 We have the next identity

$$
\begin{aligned}
K_{\widehat{X}} & +S+\sum_{j \in J_{p}} \widehat{r}^{i}\left(\tau_{k}, a_{\min }^{0}\left(\tau_{k}\right)\right) \widehat{Y}_{i}+\mu^{\star}(\widetilde{A}) \equiv \\
& \equiv \widehat{\mu}^{\star}\left(\left(1+t^{0} m_{0}\right) \widetilde{\mu}^{\star}\left(\left\{K_{X}+Y_{\tau_{k}}+A\right\}\right)-E\left(\tau_{k}, a_{\min }^{0}\left(\tau_{k}\right)\right)+K_{\widetilde{X} / X}\right)+ \\
& +\sum_{j \in J_{n}} \widehat{l}^{\imath}\left(\tau_{k}, a_{\min }^{0}\left(\tau_{k}\right)\right) E_{i}
\end{aligned}
$$

for all $k \gg 0$.

The analytic methods are now particularly useful, in the following context. By the nonvanishing theorem in [22] and its consequences (cf. sections 2 and 3), the cohomology class $\alpha\left(\tau_{k}, a_{\min }^{0}\left(\tau_{k}\right)\right)$ contains an effective $\mathbb{R}$-divisor, whose support does not contains $S$. In particular, if we denote by

$$
\widehat{\Theta}_{k} \in \alpha\left(\tau_{k}, a_{\min }^{0}\left(\tau_{k}\right)\right)
$$


a current with minimal singularities in the above class, then its restriction to $S$ denoted by $\widehat{\Theta}_{k \mid S}$ is a well-defined closed positive current in the restriction of the class.

B.4 The restriction of the current $\widehat{\Theta}_{k}$ to $S$ can be written as follows (see [25])

$$
\widehat{\Theta}_{k \mid S}:=\sum_{j \in J_{p}} \rho_{\min }^{j}\left(\tau_{k}\right)\left[\widehat{Y}_{j \mid S}\right]+\Lambda_{k, S}
$$

so the class $\alpha\left(\tau_{k}, a_{\min }^{0}\left(\tau_{k}\right)\right)-\left\{\sum_{j \in J_{p}} \min \left(\widehat{r}^{i}\left(\tau_{k}, a_{\min }^{0}\left(\tau_{k}\right)\right), \rho_{\min }^{j}\left(\tau_{k}\right)\left[\widehat{Y}_{j \mid S}\right]\right\}\right.$ is pseudoeffective.

By using the properties B.1-B.4, we will define next a set which encodes the sequence $\tau_{k}$, together with the extension data for the sections of the multiples of the bundle $K_{\widehat{X}}+S+\sum_{j \in J_{p}} \widehat{r}^{i}\left(\tau_{k}, a_{\min }^{0}\left(\tau_{k}\right) \widehat{Y}_{i}+\mu^{\star}(\widetilde{A})_{\mid S}\right.$. Next, by the induction hypothesis the set we will define will turn out to be a rational polyhedron, and this will enable us to finish the proof, and also to study the variation of the functions $\tau \rightarrow a_{\min }^{0}(\tau)$.

\section{$\S 2 . C$ The induced polytope and its properties}

We recall that our primary goal is to show that the elements of the sequence $\left(\tau_{k}\right)$ cannot be extremal points of $\mathcal{E}_{Y, A}$, as soon as $k$ is large enough. For example, it would be enough to determine $\tau_{k 0} \in \mathcal{E}_{Y, A}$ such that $\tau_{k}$ belongs to the interior of the segment $\left[\tau_{0}, \tau_{k 0}\right]$.

To this end, we will use the pseudoeffectivity criteria established in [22], in the next framework. Let $\bar{X}$ be a projective manifold, and let $\bar{S}, \bar{Y}_{j}$ be a set of strictly normal crossing hypersurfaces, such that $\bar{Y}_{j} \cap \bar{Y}_{k}=\emptyset$ if $j \neq k$. We fix a $\mathbb{Q}$-bundle $\bar{A}$ on $\bar{X}$, such that for every $\delta>0$, there exists a set $0<\delta^{j}<\delta$ of positive real numbers, such that $A-\sum_{j} \delta^{j} Y_{j}$ is ample. Then we have the next statements, which are implicit in $[22]$.

2.C.1 Theorem [22]. Let $0 \leq \nu^{j}<1$; we assume that for all $\varepsilon \ll 1$, there exists a current

$$
T_{\varepsilon} \in\left\{K_{\bar{X}}+\bar{S}+\sum_{j} \nu^{j} \bar{Y}_{j}+\bar{A}\right\}
$$

whose Lelong number along $\bar{S}$ is equal to zero, and such that $T_{\varepsilon} \geq-\varepsilon \omega$, where $\omega$ is a Kähler form on $X$. Then the class $\left\{K_{X}+\bar{S}+\sum_{j} \nu^{j} \widehat{Y}_{j}+\widehat{A}\right\}$ contains an effective $\mathbb{R}$-divisor whose support does not include $\bar{S}$.

One of the important tools in the proof of the theorem above is the statement (see [22], the paragraph $1 . \mathrm{H})$.

2.C.2 Theorem [22]. We assume that the numbers $\nu^{j}$ above are rational; there exists a positive real $\varepsilon \ll 1$ such that the following property holds true.

Any section $u \in H^{0}\left(\bar{S}, q\left(K_{\bar{X}}+\bar{S}+\sum_{j} \nu^{j} \bar{Y}_{j}+\bar{A}\right)_{\mid S}\right)$ extends to $\bar{X}$, provided that there exists $T \in\left\{K_{\bar{X}}+\bar{S}+\sum_{j} \nu^{j} \bar{Y}_{j}+\bar{A}\right\}$ a closed current whose restriction to $\bar{S}$ is well-defined, such that $T \geq-\varepsilon / q \omega$ and such that

$$
\operatorname{ord}_{\bar{Y}_{j \mid \bar{S}}}(u) \geq q \min \left(\nu\left(T_{\mid \bar{S}}, Y_{j \mid \bar{S}}\right), \nu^{j}\right)-\varepsilon
$$


for all $j$. In particular, the bundle $K_{\bar{X}}+\bar{S}+\sum_{j} \nu^{j} \bar{Y}_{j}+\bar{A}$ is (pseudo)effective, if a couple $(u, T)$ as above exists.

We refer to [3], [6], [8], [9], [12], [15], [16], [21], [26], [27], [31], [32], [33], [34] and the references therein for similar results/ideas.

Coming back to the discussion at the begining of this paragraph, we intend to use the statement above in order to determine the point $\tau_{k 0}$. The couple $(T, u)$ with the properties required by 2.C. 2 will be obtained via the theorem 0.1 in lower dimensions ; it is at this moment that the polytopes of Shokurov will play a crucial role.

The definition of the following set is modeled after the properties $\mathbf{B}_{\mathbf{j}}$ above, by decoupling the variables.

Let $C>0$ be an upper bound for the sequences $\left(a_{\min }^{0}\left(\tau_{k}\right)\right)$ and $\left(\rho_{\min }^{j}\left(\tau_{k}\right)\right)$; a finite upper bound indeed exists, thanks to the fact that the cohomology classes that contains the currents having the above singularities belong to a bounded set in $\operatorname{Psef}(X)$, respectively $\operatorname{Psef}(S)$. We introduce the following set

$$
\mathcal{E}_{\mid S}:=\left\{(\tau, a, \rho) \in[0,1]^{N} \times[0, C] \times[0, C]^{\left|J_{p}\right|}: \text { C.1 - C.4 below are satisfied }\right\}
$$

C.1 We have

$$
1-\varepsilon_{0} \geq \widehat{r}^{i}(\tau, a) \geq 0 \text { and } \widehat{l}^{i}(\tau, a) \geq 0
$$

C.2 The cohomology class of the $\mathbb{R}$-bundle

$$
D(\tau, a, \rho):=K_{S}+\sum_{j \in J_{p}}\left(\widehat{r}^{i}(\tau, a)-\rho^{i}\right)_{+} \widehat{Y}_{i}+\mu^{\star}(\widetilde{A})
$$

is pseudoeffective on $S$, where we denote by $(x)_{+}:=\max (x, 0)$;

C.3 We have the next numerical equivalence

$$
\begin{aligned}
& K_{\widehat{X}}+S+\sum_{j \in J_{p}} \widehat{r}^{i}(\tau, a) \widehat{Y}_{i}+\widehat{\mu}^{\star}(\widetilde{A}) \equiv \\
& \left.\left.\widehat{\mu}^{\star}\left(\left(1+t^{0} m_{0}\right) \widetilde{\mu}^{\star}\left(K_{X}+Y_{\tau}+A\right\}\right)-E(\tau, a)+K_{\widetilde{X} / X}\right)\right)+\sum_{j \in J_{n}} \widehat{l}^{\imath}(\tau, a) E_{i}
\end{aligned}
$$

(we use the notation in (34)) ;

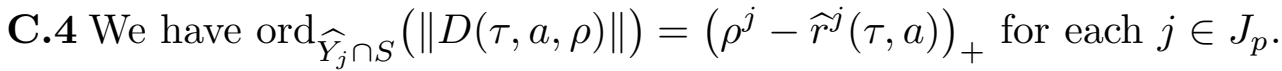

Concerning the conditions above, our first claim is that the set $\mathcal{E}_{1}$ defined just by the conditions C.1 - C.3 is a rational polytope. Indeed, the requirements C.1 and C.3 are affine (in)equations, and the linear forms defining them have rational coefficients. As for the condition C.2, we consider the set

$$
\mathcal{E}_{\widehat{W}, \widehat{\mu}^{\star}(\widetilde{A})}:=\left\{\eta=\left(\eta_{j}\right) \in\left[0,1-\varepsilon_{0}\right]^{\left|J_{p}\right|}: K_{S}+\sum_{j \in J_{p}} \eta^{j} \widehat{Y}_{i}+\mu^{\star}(\widetilde{A}) \in \operatorname{Psef}(S)\right\}
$$


The induction hypothesis, together with the simple considerations at the end of the section 1 show that $\mathcal{E}_{\widehat{W}, \widehat{\mu}^{\star}(\widetilde{A})}$ is indeed a rational polytope, and then the condition C.3 reads as

$$
\left(\widehat{r}^{i}(\tau, a)-\rho^{i}\right)_{+} \in \mathcal{E}_{\widehat{W}, \widehat{\mu^{\star}}(\widetilde{A})},
$$

and thus the set $\mathcal{E}_{1}$ is a rational polytope ; we fix its vertices $\left(\tau_{j}, a_{j}, \rho_{j}\right)_{j=1, \ldots, G}$, and let $d$ be its dimension.

For each $k=1, \ldots, G$, we define $D_{k}:=D\left(\tau_{k}, a_{k}, \rho_{k}\right)$. It is a pseudoeffective $\mathbb{Q}$-line bundle, and therefore it is effective, by the non-vanishing theorem [2], [22]. The Cox ring associated to $\left(D_{k}\right)$ is finitely generated, by induction, and therefore for each $\widehat{Y}_{j \mid S}$ the associated asymptotic vanishing order function is piecewise affine, by the result [11] recalled in the section 1 .

We consider a decomposition of $\mathcal{E}_{1}=\bigcup_{k=1}^{M} \mathcal{C}_{k}$ into standard simplexes, such that the functions below

$$
(\tau, a, \rho) \rightarrow\left(\widehat{r}^{i}(\tau, a)-\rho^{i}\right)_{+}
$$

and

$$
(\tau, a, \rho) \rightarrow \operatorname{ord}_{\widehat{Y}_{j} \cap S}(\|D(\tau, a, \rho)\|)
$$

becomes affine when restricted to any $\mathcal{C}_{k}$; we remark that the existence of such a decomposition is slightly different from the assertion in the statement 1.2 , but it can be seen to follow, by a quick linear algebra argument. Thus, the equation C.4 imposes affine requirements on the parameters $(\tau, a, \rho)$, and in conclusion, we have just proved the next statement.

2.C.3 Lemma. The set $\mathcal{E}_{\mid S}$ defined by the relation $\mathbf{C . 1}-\mathbf{C . 4}$ above is a rational polytope.

We stress on the fact that the extension of the function "ord" to the $\mathbb{R}$-divisors we are using in $\mathbf{C . 4}$ depends on the polytope $\mathcal{E}_{1}$; hopefully, this will not cause too much confusion.

We denote by $\left(\tau_{j}, a_{j}, \rho_{j}\right)_{j \in K}$ the vertices of $\mathcal{E}_{\mid S}$; they have rational coordinates, and for the purposes of the next corollary we can assume that they are independent in the affine sense- this can always be achieved modulo a subdivision.

Given a point $(\tau, a, \rho) \in \mathcal{E}_{\mid S}$, we can write it as follows

$$
(\tau, a, \rho)=\sum_{j \in K} \lambda^{j}\left(\tau_{j}, a_{j}, \rho_{j}\right)
$$

where $\sum_{j} \lambda_{j}=1$; moreover, if the coordinates of the vector above are rational, then its affine coordinates $\left(\lambda_{j}\right)$ are rational as well.

The main use of the linear structure of the set $\mathcal{E}_{\mid S}$ is revealed by the next statement, which can be seen as a uniform non-vanishing, see also [2], [3]. 
2.C.4 Proposition. There exists an integer $q_{0} \in \mathbb{Z}_{+}$with the following property : let

$$
(\tau, a, \rho) \in \mathcal{E}_{\mid S}
$$

be a point with rational coordinates, and let $q \in \mathbb{Z}_{+}$be a common denominator of its affine coordinates $\left(\lambda_{j}\right)$ in the equality (44). Then the bundle

$$
q q_{0}\left(K_{S}+\sum_{j \in J_{p}} \widehat{r}^{j}(\tau, a) \widehat{Y}_{i \mid S}+\mu^{\star}(\widetilde{A})\right)
$$

has a section whose vanishing order along the set $\widehat{Y}_{i \mid S}$ is exactly $q q_{0} \rho^{i}$.

Proof. We consider the $\mathbb{Q}$-bundle $D\left(\tau_{j}, a_{j}, \rho_{j}\right)$ corresponding to a vertex of $\mathcal{E}_{\mid S}$. Its asymptotic vanishing order at the generic point of $\widehat{Y}_{i \mid S}$ is given by the condition C.4, that is to say $\operatorname{ord}_{\widehat{Y}_{i} \cap S}\left(\| D\left(\tau_{j}, a_{j}, \rho_{j} \|\right)=\left(\rho^{i}-\widehat{r}^{j}\left(\tau_{j}, a_{j}\right)\right)_{+}\right.$. Since by induction we already know that the ring associated to $D\left(\tau_{j}, a_{j}, \rho_{j}\right)$ is finitely generated, we obtain a section $u_{j}$ of the bundle $q_{j} D\left(\tau_{j}, a_{j}, \rho_{j}\right)$ such whose vanishing order on $\widehat{Y}_{i \mid S}$ is equal to $q_{j}\left(\rho^{i}-\widehat{r}^{i}\left(\tau_{j}, a_{j}\right)\right)_{+}$, for each $i \in J_{p}$. Then we obtain -by twisting with an appropriate divisor- a section

$$
v_{j} \in H^{0}\left(S, q_{j}\left(K_{S}+\sum_{k \in J_{p}} \widehat{r}^{k}\left(\tau_{j}, a_{j}\right) \widehat{Y}_{k \mid S}+\mu^{\star}(\widetilde{A})\right)\right)
$$

whose vanishing order at the generic point of $\widehat{Y}_{i \mid S}$ is equal to $q_{j} \rho^{i}$, for each $i \in J_{p}$. The section we seek is obtained by convex combination of the $v_{j}$, so the proposition is proved.

The same argument shows that for any point $(\tau, a, \rho) \in \mathcal{E}_{\mid S}$, we can construct an effective $\mathbb{R}$-divisor $\Xi \in\left\{K_{S}+\sum_{k \in J_{p}} \widehat{r}^{k}(\tau, a) \widehat{Y}_{k \mid S}+\mu^{\star}(\widetilde{A})\right\}$ whose vanishing order along $\widehat{Y}_{m \mid S}$ is equal to $\rho^{m}$, for every $m \in J_{n}$.

Our next goal is to show that we have $\left(\tau_{k}, a_{\text {min }}^{0}\left(\tau_{k}\right), \rho_{\min }\left(\tau_{k}\right)\right) \in \mathcal{E}_{\mid S}$; this will be the consequence of a more general result. Before stating it, we introduce one more notation.

Let

$$
\mathcal{G} \subset[0,1]^{N} \times[0, C]
$$

(where $C$ is the constant fixed at the begining of this paragraph) be the set described by the following three conditions.

C.5 We have $0 \leq \widehat{r}^{i}(\tau, a) \leq 1-\varepsilon_{0}$ for all $i \in J_{p}$;

C.6 The class $\left\{K_{\widehat{X}}+S+\sum_{i \in J_{p}} \widehat{r}^{i}(\tau, a) \widehat{Y}_{i}+\widehat{\mu}^{\star}(\widetilde{A})\right\}$ is pseudoeffective.

C.7 We denote by $\widehat{\Theta}(\tau, a) \in\left\{K_{\widehat{X}}+S+\sum_{j \in J_{p}} \widehat{r}^{i}(\tau, a) \widehat{Y}_{i}+\widehat{\mu}^{\star}(\widetilde{A})\right\}$ the current with minimal singularities ; then we have $\nu\left(\Theta_{\min }(\tau, a), S\right)=0$. 
An important remark is that the set $\mathcal{G}$ is compact and convex : the fact that it is convex is immediate from its definition, whereas its closeness is a direct consequence of the theorem 2.C.1.

As it was recalled just before the property B.4 above, the restriction $\widehat{\Theta}(\tau, a)_{\mid S}$ is well defined, and we decompose the restriction current as follows

$$
\widehat{\Theta}(\tau, a)_{\mid S}=\sum_{j \in J_{p}} \rho_{\min }^{i}(\tau, a) \widehat{Y}_{i \mid S}+\Lambda_{S}(\tau, a)
$$

where the Lelong number of $\Lambda_{S}(\tau, a)$ on each $Y_{j \mid S}$ is equal to zero. Therefore, by the previous equality we define the function

$$
\rho_{\min }: \mathcal{G} \rightarrow \mathbb{R}_{+}^{\left|J_{p}\right|}
$$

if $\tau \in \mathcal{E}_{Y, A}$ is an element such that $\left(\tau, a_{\min }^{0}(\tau)\right) \in \mathcal{G}$, then we use the notation

$$
\rho_{\min }^{i}(\tau):=\rho_{\min }^{i}\left(\tau, a_{\min }^{0}(\tau)\right)
$$

and we remark that this is consistent with the notations in B.4 for $\tau=\tau_{k}$, an element of our initial sequence.

If the point $(\tau, a) \in \mathcal{G}$ has rational coordinates, then by the results in [22] we know that $\rho_{\text {min }}^{j}(\tau, a) \in \mathbb{Q}$, for any $j \in J_{p}$. An important result of this subsection is the following statement.

2.C.5 Proposition. We assume that the requirements C.1 and C.3 are fulfilled by some element $(\tau, a) \in \mathcal{G} \cap \mathbb{Q}^{1+N}$. Then the point $\left(\tau, a, \rho_{\min }(\tau, a)\right)$ belongs to the polytope $\mathcal{E}_{\mid S}$. In particular, we have $\left(\tau_{k}, a_{\min }^{0}\left(\tau_{k}\right), \rho_{\min }\left(\tau_{k}\right)\right) \in \mathcal{E}_{\mid S}$ for all $k \in \mathbb{Z}_{+}$such that the coordinates of $\tau_{k}$ are rational.

Proof. By the relation (46), we obtain

$$
\sum_{j \in J_{p}} \rho_{\min }^{i}(\tau, a) \widehat{Y}_{i \mid S}+\Lambda_{S}(\tau, a) \equiv K_{S}+\sum_{j \in J_{p}} \widehat{r}^{i}(\tau, a) \widehat{Y}_{i \mid S}+\widehat{\mu}^{\star}(\widetilde{A})
$$

and therefore we have

$$
\begin{aligned}
\sum_{j \in J_{p}}\left(\rho_{\min }^{i}(\tau, a)-\widehat{r}^{i}(\tau, a)\right)_{+} \widehat{Y}_{i \mid S}+\Lambda_{S}(\tau, a) & \equiv K_{S}+\sum_{j \in J_{p}}\left(\widehat{r}^{i}(\tau, a)-\rho_{\min }^{i}(\tau, a)\right)_{+} \widehat{Y}_{i \mid S}+ \\
& +\widehat{\mu}^{\star}(\widetilde{A}) .
\end{aligned}
$$

In conclusion, the $\mathbb{Q}$-bundle $D\left(\tau, a, \rho_{\min }(\tau, a)\right)$ is pseudoeffective, and this proves that the point $\left(\tau, a, \rho_{\min }(\tau, a)\right)$ verifies the condition C.2.

Let $u_{0}$ be a section of the bundle

$$
q\left(K_{S}+\sum_{i \in J_{p}}\left(\widehat{r}^{i}(\tau, a)-\rho_{\min }^{i}(\tau, a)\right)_{+} \widehat{Y}_{i \mid S}+\mu^{\star}(\widetilde{A})\right)
$$


such an object defines canonically a section $u$ of the bundle

$$
q\left(K_{S}+\sum_{i \in J_{p}} \widehat{r}^{i}(\tau, a) \widehat{Y}_{i \mid S}+\mu^{\star}(\widetilde{A})\right)
$$

whose zero set contains the divisor $\left.\sum_{i \in J_{p}} \min \left\{\rho_{\min }^{i}(\tau, a), \widehat{r}^{i}(\tau, a)\right)\right\} \widehat{Y}_{i \mid S}$. But then the section $u$ admits an extension to $\widehat{X}$, by 2.C.2 (see also [1], [12], [14]), so in particular we have

$$
\operatorname{ord}_{\widehat{Y}_{j} \cap S}\left(\left\|D\left(\tau, a, \rho_{\min }(\tau, a)\right)\right\|\right) \geq\left(\rho_{\min }^{j}(\tau, a)-\widehat{r}^{j}(\tau, a)\right)_{+}
$$

for all $j \in J$, because the metric induced by the extension of $u$ is more singular than the metric with minimal singularities of the corresponding bundle.

The ring of sections associated to $D\left(\tau, a, \rho_{\min }(\tau, a)\right)$ is finitely generated by induction, therefore we have

$$
\operatorname{ord}_{\widehat{Y}_{j} \cap S}\left(\left\|D\left(\tau, a, \rho_{\min }(\tau, a)\right)\right\|\right) \nu\left(\Theta_{\sigma}(\tau, a), \widehat{Y_{j}} \cap S\right)
$$

where we denote by $\Theta_{\sigma}(\tau, a)$ the current associated to the algebra of sections of the bundle $D\left(\tau, a, \rho_{\min }(\tau, a)\right)$, i.e. the current with minimal singularities in the sense of Siu, whose definition was recalled in the paragraph 1.

Next, the closed positive current

$$
\widehat{\Theta}(\tau, a)_{\mid S}-\sum_{i \in J_{p}} \min \left\{\rho_{\min }^{i}(\tau, a), \widehat{r}^{i}(\tau, a)\right\}\left[\widehat{Y}_{i \mid S}\right] \in\left\{D\left(\tau, a, \rho_{\min }(\tau, a)\right)\right\}
$$

is certainly more singular than the current with minimal singularities of the class above.

Summing up the previous considerations, we have shown that the quantity we are interested in $\left(\rho_{\min }^{j}(\tau, a)-\widehat{r}^{j}(\tau, a)\right.$ is smaller than the generic Lelong number of the current $\Theta_{\sigma}(\tau, a)$ on $\widehat{Y_{j}} \cap S$, and greater than the generic Lelong number of the current with minimal singularities of the class $\left\{D\left(\tau, a, \rho_{\min }(\tau, a)\right)\right\}$ along $\widehat{Y_{j}} \cap S$.

Since the bundle $D\left(\tau, a, \rho_{\min }(\tau, a)\right)$ can be written as $K_{S}+L_{k}$, where $L_{k}$ is big and klt, the current with minimal singularities in the sense of Siu coincides with the current with minimal singularities in the sense of Demailly, as it was established in [22] ; in conclusion, the relation (48) becomes an equality, and 2.C.5 is proved.

Remark. Following the articles [14], [19], one can avoid the use of the closed positive currents in the definition of the coefficients $\rho_{\min }^{j}(\tau, a)$. Nevertheless, we prefer the approach explained above, since the notion of metric with minimal singularities is meaningful in a very general context, as soon as the cohomology class in question is pseudoeffective.

We will assume from now on that each $\tau_{k}$ has rational coordinates, unless explicitly specified otherwise. The sequence $\left(\tau_{k}\right)$ converges to the limit $\tau_{0}$ for which no rationality assumption is made. 
The following corollary is similar to the results established in [19], and can be seen as a consequence of the preceding arguments, together with elementary convex geometry considerations. The main idea of the proof goes back at least to [14] (see the paragraph concerning the rationality of the restricted algebras ; see equally [22] for a use of this idea in analytic setting).

2.C.6 Corollary. Up to the choice of a subsequence, for all $k \gg 0$, there exists an element $\tau_{k 0} \in \mathcal{E}_{Y, A}$ such that:

(i) The point $\tau_{k}$ belongs to the interior of the segment $\left[\tau_{0}, \tau_{k 0}\right]$;

(ii) The restriction of the function $\tau \rightarrow a_{\min }^{0}(\tau)$ to the segment $\left[\tau_{0}, \tau_{k 0}\right]$ is affine.

(iii) For each $\tau \in\left[\tau_{0}, \tau_{k 0}\right]$, we have $\left(\tau, a_{\min }^{0}(\tau)\right) \in \mathcal{G}$, and the functions $\tau \rightarrow \rho_{\min }^{j}(\tau)$ defined in $(47)$ are affine on $\left[\tau_{0}, \tau_{k 0}\right]$.

Proof. By the proposition 2.C.5 we have $\left(\tau_{k}, a_{\min }^{0}\left(\tau_{k}\right), \rho_{\min }\left(\tau_{k}\right)\right) \in \mathcal{E}_{\mid S}$, for any $k \gg 0$; again, we can assume that the vertices $\left(\tau_{j}, a_{j}, \rho_{j}\right)_{j=1, \ldots, d+1}$ of $\mathcal{E}_{\mid S}$ are affinely independent (by passing to a subdivision of the polytope if necessary).

The affine coordinates of the point $\left(\tau_{k}, a_{\min }^{0}\left(\tau_{k}\right), \rho_{\min }\left(\tau_{k}\right)\right)$ are written as follows

$$
\left(\tau_{k}, a_{\min }^{0}\left(\tau_{k}\right), \rho_{\min }\left(\tau_{k}\right)\right)=\sum_{j=1}^{d+1} \lambda_{k}^{j}\left(\tau_{j}, a_{j}, \rho_{j}\right),
$$

where $\lambda_{k}^{j}$ are positive rational numbers, such that $\sum_{j} \lambda_{k}^{j}=1$. For each index $j$, we can assume that $\lim _{k \rightarrow \infty} \lambda_{k}^{j}:=\lambda_{0}^{j}$, and we consider the corresponding point

$$
\left(\tau_{0}, a_{0}, \rho_{0}\right)=\sum_{j=1}^{d+1} \lambda_{0}^{j}\left(\tau_{j}, a_{j}, \rho_{j}\right) \in \mathcal{E}_{\mid S},
$$

where the first component is indeed $\tau_{0}$, since $\tau_{k} \rightarrow \tau_{0}$.

The function $\tau \rightarrow a_{\text {min }}^{0}(\tau)$ defined on $\mathcal{E}_{Y, A}$ is convex and bounded, therefore we have

$$
a_{0} \leq a_{\min }^{0}\left(\tau_{0}\right)
$$

Next, due to the possible non-rationality of the coordinates of $V_{0}:=\left(\lambda_{0}^{j}\right)$, we will use a few classical results from diophantine approximation theory (see [2], [19], [22] for similar considerations).

Let $\eta>0$ be a positive real ; then there exists a finite set of vectors $V_{\eta s}=\left(\lambda_{\eta s}^{j}\right)$ such that :

A.1 We have $\sum_{j} \lambda_{\eta s}^{j}=1$, and moreover the $\lambda_{\eta s}^{j}$ are positive rational numbers ;

A.2 For each $s$, we have $q_{\eta s}\left\|V_{0}-V_{\eta s}\right\| \leq \eta$, where $q_{\eta s}$ is a positive integer, such that $q_{\eta s} \lambda_{\eta s}^{j} \in \mathbb{Z}_{+}$, for each $j$

A.3 We have $V_{0}=\sum_{s} \alpha_{s} V_{\eta s}$, where $\alpha_{p}$ are positive real numbers, such that $\sum_{s} \alpha_{s}=1$.

A.4 For each $s$, the point $\tau_{\eta s}:=\sum_{i} \lambda_{\eta s}^{i} \tau_{i}$ belongs to the minimal rational affine subspace $A\left(\tau_{0}\right) \subset \mathbb{R}^{N}$ which contains $\tau_{0}$. 
We use the coordinates of the vectors $V_{\eta s}$ in order to define a point in the polytope $\mathcal{E}_{\mid S}$, as follows

$$
\left(\tau_{\eta s}, a_{\eta s}, \rho_{\eta s}\right):=\sum_{j=1}^{d+1} \lambda_{\eta s}^{j}\left(\tau_{j}, a_{j}, \rho_{j}\right),
$$

By the proposition 2.C.4, there exists a section $u_{\eta s}$ of the bundle

$$
q_{0} q_{\eta s}\left(K_{S}+\sum_{j \in J_{p}}\left(\widehat{r}^{i}\left(\tau_{\eta s}, a_{\eta s}\right) \widehat{Y}_{i \mid S}+\mu^{\star}(\widetilde{A})\right)\right.
$$

whose vanishing order on $\widehat{Y}_{i \mid S}$ is precisely $q_{0} q_{\eta s} \rho_{\eta s}^{i}$.

The theorem 2.C.2 implies that the section $u_{\eta s}$ admits an extension to $\widehat{X}$. Indeed, the current $T$ in 2.C.2 is obtained by moving the current $\widehat{\Theta}_{k}$ (see B.4) into the class $\left\{K_{X}+S+\sum_{i \in J_{p}}\left(\widehat{r}^{i}\left(\tau_{\eta s}, a_{\eta s}\right) \widehat{Y}_{i}+\mu^{\star}(\widetilde{A})\right\}\right.$; the loss of positivity induced by this operation is of order $\mathcal{O}\left(\left\|\tau_{k}-\tau_{\eta s}\right\|+\left|a_{\text {min }}^{0}\left(\tau_{k}\right)-a_{\eta s}\right|\right)$. We equally have the inequality $q_{\eta s}\left|\rho_{\eta s}^{i}-\rho_{\min }^{i}\left(\tau_{k}\right)\right| \leq C \eta$ provided that $k \geq k_{\eta}$, and therefore the assertion A.2 together with the relation

$$
\left(\tau_{k}, a_{\min }^{0}\left(\tau_{k}\right), \rho_{\min }\left(\tau_{k}\right)\right) \rightarrow\left(\tau_{0}, a_{0}, \rho_{0}\right)=\sum_{j=1}^{d+1} \lambda_{0}^{j}\left(\tau_{j}, a_{j}, \rho_{j}\right)
$$

shows that the hypothesis of 2.C.2 are satisfied as soon as $\eta$ is small enough and $k$ is large enough.

Therefore, for all indexes $s$ we have $\left(\tau_{\eta s}, a_{\eta s}\right) \in \mathcal{G}$ provided that $\eta \ll 1$; it follows that the divisor of zeroes of $u_{\eta s}$ is greater than

$$
q_{0} q_{\eta s} \sum_{j \in J_{p}} \rho_{\min }^{j}\left(\tau_{\eta s}, a_{\eta s}\right)\left[\widehat{Y}_{j \mid S}\right]
$$

and this translates as

$$
\rho_{\eta s}^{i} \geq \rho_{\min }^{i}\left(\tau_{\eta s}, a_{\eta s}\right)
$$

for every $i \in J_{p}$.

Furthermore, we have $\left(\tau_{\eta s}, a_{\eta s}, \rho_{\eta s}\right) \in \mathcal{E}_{\mid S}$ and thus the numerical relation C.3 shows that the extension of the section $u_{\eta s}$ to $\widehat{X}$ induces a closed positive current in the class $\left\{K_{X}+Y_{\tau_{\eta s}}+A\right\}$, whose Lelong number along $S$ is equal to $a_{\eta s}$; in particular, we have $\tau_{\eta s} \in \mathcal{E}_{Y, A}$ and moreover

$$
a_{\eta s} \geq a_{\min }^{0}\left(\tau_{\eta s}\right)
$$

By combining (49), (51) and A.3 we infer that

$$
a_{\min }^{0}\left(\tau_{0}\right) \geq a_{0} \geq \sum \alpha_{s} a_{\min }^{0}\left(\tau_{\eta s}\right)
$$


and therefore we have

$$
a_{\eta s}=a_{\min }^{0}\left(\tau_{\eta s}\right), \quad a_{\min }^{0}\left(\tau_{0}\right)=\sum \alpha_{s} a_{\min }^{0}\left(\tau_{\eta s}\right)
$$

thanks to the convexity of the function $a_{\mathrm{min}}^{0}$; we equally have

$$
\rho_{0}^{i} \geq \sum_{s} \alpha_{s} \rho_{\min }^{i}\left(\tau_{\eta s}, a_{\eta s}\right)
$$

For further use, we formulate next some conclusions of the arguments provided so far.

(a) We have $a_{\eta s}=a_{\min }^{0}\left(\tau_{\eta s}\right)$, as stated in (52).

(b) If $\tau \in A\left(\tau_{0}\right)$ belongs to the convex hull $\mathcal{C}_{\eta}$ of $\left(\tau_{\eta s}\right)$, then $\tau \in \mathcal{E}_{Y, A}$; in addition, the relation (52) implies that the function $\tau \rightarrow a_{\min }^{0}(\tau)$ is affine on $\mathcal{C}_{\eta}$. This is an immediate consequence of the relation (52), together with elementary convexity arguments.

(c) For each $\tau \in \mathcal{C}_{\eta}$, we have $\left(\tau, a_{\min }^{0}(\tau)\right) \in \mathcal{G}$, and moreover, the function $\tau \rightarrow \rho_{\min }(\tau)$ is affine on a small open set $\Omega \subset \mathcal{C}_{\eta}$ containing the point $\tau_{0}$. Indeed, by the property (a) we obtain $\left(\tau_{\eta s}, a_{\min }^{0}\left(\tau_{\eta s}\right)\right) \in \mathcal{G}$ for all the indexes $s$, and combined with the fact that the function $a_{\min }^{0}$ is affine on $\mathcal{C}_{\eta}$, this shows the first part of (c), plus the convexity of the functions $\tau \rightarrow \rho_{\min }^{j}(\tau)$ on the set $\mathcal{C}_{\eta}$. The next observation is that we have $\left(\tau_{0}, a_{\text {min }}^{0}\left(\tau_{0}\right), \rho_{\min }\left(\tau_{0}\right)\right) \in \mathcal{E}_{\mid S}$ as a consequence of the proposition 2.C.5. Finally, the fact that this functions are locally affine near $\tau_{0}$ is obtained by using the diophantime approximation procedure A.1-A.4 described above for the point $\left(\tau_{0}, a_{\min }^{0}\left(\tau_{0}\right), \rho_{\min }\left(\tau_{0}\right)\right)$.

Next, we introduce the notation $V_{k}:=\left(\lambda_{k}^{j}\right)$, and we consider the vector $q_{\eta s}\left(V_{\eta s}-V_{k}\right)$. Its norm is smaller than $2 \eta$, provided that $k \geq k_{\eta}$ is large enough. But then there exists a positive integer $q_{\eta s}^{\prime}$ and of a vector $V_{\eta s}^{\prime}$ such that the coordinates of $q_{\eta s}^{\prime} V_{\eta s}^{\prime}$ are integers, and such that we have

$$
q_{\eta s}\left(V_{\eta s}-V_{k}\right)=q_{\eta s}^{\prime}\left(V_{k}-V_{\eta s}^{\prime}\right)
$$

(see e.g. [2], or "any good book on diophantine approximation"). The relation (54) together with the fact that the coordinates of $q_{\eta s}^{\prime} V_{\eta s}^{\prime}$ are integers implies in particular that the coordinates of $V_{\eta s}^{\prime}$ are positive rational numbers, and that their sum is equal to 1 .

Then we consider the vector

$$
\left(\tau_{\eta s}^{\prime}, a_{\eta s}^{\prime}, \rho_{\eta s}^{\prime}\right):=\sum_{j \in I_{1}} \lambda_{\eta s}^{\prime j}\left(\tau_{j}, a_{j}, \rho_{j}\right)
$$

where $\left(\lambda_{\eta s}^{\prime j}\right)$ are the coordinates of $V_{\eta s}^{\prime}$. The same extension arguments which were used a few lines above for $\left(\tau_{\eta s}, a_{\eta s}, \rho_{\eta s}\right)$ will show in the first place that $\left(\tau_{\eta s}^{\prime}, a_{\eta s}^{\prime}\right) \in \mathcal{G}$. We also obtain that $K_{X}+Y_{\tau_{\eta s}^{\prime}}+A \in \operatorname{Psef}(X)$ together with

$$
a_{\eta s}^{\prime} \geq a_{\min }^{0}\left(\tau_{\eta s}^{\prime}\right)
$$


and

$$
\rho_{\eta s}^{\prime j} \geq \rho_{\min }^{j}\left(\tau_{\eta s}^{\prime}, a_{\eta s}^{\prime}\right)
$$

The relation (54) implies that

$$
V_{k}=\frac{q_{\eta s}}{q_{\eta s}+q_{\eta s}^{\prime}} V_{\eta s}+\frac{q_{\eta s}^{\prime}}{q_{\eta s}+q_{\eta s}^{\prime}} V_{\eta s}^{\prime}
$$

for every $s$, and therefore we obtain

$$
\left(\tau_{k}, a_{\min }^{0}\left(\tau_{k}\right), \rho_{\min }\left(\tau_{k}\right)\right)=\frac{q_{\eta s}}{q_{\eta s}+q_{\eta s}^{\prime}}\left(\tau_{\eta s}, a_{\eta s}, \rho_{\eta s}\right)+\frac{q_{\eta s}^{\prime}}{q_{\eta s}+q_{\eta s}^{\prime}}\left(\tau_{\eta s}^{\prime}, a_{\eta s}^{\prime}, \rho_{\eta s}^{\prime}\right) .
$$

The convexity of the function $a_{\mathrm{min}}^{0}$, together with the relations (52) and (56) shows that this function is in fact affine on the segment $\left[\tau_{\eta s}, \tau_{\eta s}^{\prime}\right]$, and that we have $a_{\eta s}^{\prime}=a_{\text {min }}^{0}\left(\tau_{\eta s}^{\prime}\right)$. As in the claim (c) above, this implies in the first place that the functions

$$
\tau \rightarrow \rho_{\min }^{j}(\tau)
$$

are convex on the segment $\left[\tau_{\eta s}, \tau_{\eta s}^{\prime}\right]$. When combined with the inequalities $(50),(57)$ and with the relation (58), we obtain that they are affine on the segment $\left[\tau_{\eta s}, \tau_{\eta s}^{\prime}\right]$, for every $s$.

The obvious consequence of these considerations is the existence of a point $\tau_{k 0} \in$ $\mathcal{E}_{Y, A}$, such that $\tau_{k}$ belongs to the interior of segment $\left[\tau_{0}, \tau_{k 0}\right]$, and for which the properties (ii) and (iii) in the statement 2.C.6 holds. Indeed, we obtain $\tau_{k 0}$ as a convex combination of points lying on the segments $\left[\tau_{\eta s}, \tau_{\eta s}^{\prime}\right]$; we do not provide further details, but nevertheless the corollary is completely proved.

In conclusion, the proof of the first point of 0.1 is finished ; we derive next some easy applications of the preceding techniques/results.

We consider the following data (similar to the set-up in the introduction). Let $\mathcal{L} \subset \mathbb{R}^{r}$ be a rational polytope, and for let $l^{j}: \mathbb{R}^{r} \rightarrow \mathbb{R}$ be a set of affine forms defined over $\mathbb{Q}$, where $j=2, \ldots N$. We assume that $0 \leq l^{j}(\theta) \leq 1-\varepsilon_{0}$, for all $\theta \in$ $\mathcal{L}$, where $\varepsilon_{0}$ is a positive real number. If the point $\tau:=\left(1, l^{2}(\theta), \ldots, l^{N}(\theta)\right)$ belongs to $\mathcal{E}_{Y, A}$, then we denote by $T(\theta)$ the current with minimal singularities in the class $K_{X}+Y_{1}+\sum_{j=2}^{N} l^{j}(\theta) Y_{j}+A$. We have the next statement (cf. [19], and implicitly [2]), which will be useful in the next paragraph.

\section{C.7 Corollary.}

a) We consider the set

$$
\mathcal{E}^{1}:=\left\{\theta \in \mathcal{L}:\left(1, l^{2}(\theta), \ldots, l^{N}(\theta)\right) \in \mathcal{E}_{Y, A}, \quad \nu\left(T(\theta), Y_{1}\right)=0\right\}
$$

Then $\mathcal{E}^{1}$ is a rational polytope. 
b) We assume furthermore that the hypersurfaces $\left(Y_{j}\right)_{j \neq 1}$ are mutually disjoint, and that $A$ is not necessarily ample, but it has the next property : for each $\delta>0$, there exists $\delta>\varepsilon^{j}>0$ such that $A-\sum_{i \neq 1} \varepsilon^{i} Y_{i}$ is ample. As in (46), we define the functions $\theta \rightarrow \rho_{\min }^{j}(\theta)$ by the restriction of $T(\theta)$ to $Y_{1}$; then $\rho_{\min }^{j}$ are piecewise affine on $\mathcal{E}^{1}$.

Proof. The part a) of the preceding statement is a direct consequence of the just finished proof of 0.1 .

Indeed, $\mathcal{E}^{1}$ above is a renamed version of the set $\mathcal{G}$ which was considered page 21-22, so in the first place it is closed. The analysis of its extremal points is carried out as above, without any modification ; in fact, it is much simpler, since we already have the (analogue of the) set $S$ above. The substitute for the polytope $\mathcal{E}_{\mid S}$-previously constructed by using of the sequence $\left(\tau_{k}\right)$ - is defined as follows.

We denote by $\mathcal{E}_{\mid Y_{1}}^{1}$ the set of elements $(\theta, \rho) \in \mathcal{E}^{1} \times[0, C]^{N-1}$ such that :

- The class $\alpha(\theta, \rho):=\left\{K_{Y_{1}}+\sum_{j=2}^{N}\left(l^{j}(\theta)-\rho^{j}\right)_{+} Y_{j \mid Y_{1}}+A\right\}$ is pseudoeffective ;

- We have $\operatorname{ord}_{Y_{j} \cap Y_{1}}(\|\alpha(\theta, \rho)\|)=\left(\rho^{j}-l^{j}(\theta)\right)_{+}$.

As in 2.C.3 we verify that $\mathcal{E}_{\mid Y_{1}}^{1}$ is a rational polytope, which contains the points $\left(q, \rho_{\min }(q)\right)$ for any $q \in \mathcal{E}^{1} \cap \mathbb{Q}^{r}$, see 2.C.4. We conclude by the (analogue of the) corollary 2.C.6.

For the proof of the part b), the polytope structure of $\mathcal{E}^{1}$ will be crucial.

Let $\theta \in \mathcal{E}^{1}$ be an arbitrary point. We consider the intersection of $\mathcal{E}^{1}$ with the minimal affine subspace in $\mathbb{R}^{r}$ defined over $\mathbb{Q}$, which contains $\theta$; this rational polytope is denoted by $A(\theta) \subset \mathcal{E}^{1}$. Since $\left(q, \rho_{\min }(q)\right) \in \mathcal{E}_{\mid Y_{1}}^{1}$ for any rational point $q \in A(\theta)$ we deduce that $\left(\theta, \rho_{\min }^{j}(\theta)\right) \in \mathcal{E}_{\mid Y_{1}}^{1}$, by a limit argument.

Next, we remark that for an arbitrary sequence $\left(\theta_{k}\right) \subset \mathcal{E}^{1}$ converging to $\theta_{0}$ such that $\theta_{k} \neq \theta_{0}$ there exists a sequence $\theta_{0 k} \subset \mathcal{E}^{1}$ such that $\theta_{k}$ belongs to the interior of the segment $\left[\theta_{0}, \theta_{0 k}\right]$ and moreover the restriction of the function $\rho_{\min }^{j}$ to the above segment is affine (again, modulo the choice of a subsequence). This claim was already verified in 2.C.6 if the coordinates of the points $\theta_{k}$ are rational ; we remark that the only use of the rationality was to infer that

$$
\left(\theta_{k}, \rho_{\min }\left(\theta_{k}\right)\right) \in \mathcal{E}_{\mid Y_{1}}^{1}
$$

The relation (58) was shown to hold a few lines ago regardless to the rationality of the components of $\theta_{k}$, thanks to the polytope structure of $\mathcal{E}^{1}$. The claim concerning the restriction of $\rho_{\min }^{j}$ to a segment $\left[\theta_{0}, \theta_{0 k}\right]$ follows as in the proof of 2.C.6 above, up to simple modifications which will not be detailed here. Another consequence of these arguments is the continuity of the functions $\rho_{\text {min }}$ up to the boundary of $\mathcal{E}^{1}$.

Let $f: \mathcal{E}^{1} \rightarrow[0, C]$ be one of the functions $\rho_{\text {min }}^{j}$ we are interested in ; it is continuous and convex, by the preceding considerations. We consider the epigraph of $f$, defined as

$$
\operatorname{Epi}(f):=\left\{(v, y) \in \mathcal{E}^{1} \times[0, C]: y \geq f(v)\right\} .
$$


We have just proved that for any sequence of points $\left(\theta_{k}\right) \subset \mathcal{E}^{1}$ converging to $\theta_{0}$, there exists a $k_{0} \gg 0$ such that for any $k \geq k_{0}$, the restriction of $f$ to a segment $\left[\theta_{0}, \theta_{k 0}\right]$ containing $\theta_{k}$ in its interior is affine. This implies that $\operatorname{Epi}(f)$ can only have finitely many extremal points on the graph of $f$, so it follows that $\operatorname{Epi}(f)$ is a polytope (the set $\operatorname{Epi}(f)$ is closed, thanks to the continuity of $f)$. This in turn is equivalent to the fact that the function $f$ is polyhedral (or piecewise affine), which is what we wanted to prove.

We note that there exists a subdivision of $\mathcal{E}^{1}$ into rational polytopes say $\mathcal{C}_{k}$, such that the restriction of $\rho_{\min }$ to each $\mathcal{C}_{k}$ is affine. The rationality assertion is a consequence of the fact that the function $\rho_{\text {min }}$ is locally affine when restricted to the minimal rational affine space containing an arbitrary point $\theta_{0}$.

\section{$\S 3$ Proof of (ii)}

The approach we will follow for the second part of the theorem 0.1 is somehow similar to the arguments presented in [14]. We recall the next notation

$$
\mathcal{A}_{r}(X):=\bigoplus_{(m, \theta) \in \Gamma_{d}} H^{0}\left(X, k\left(K_{X}+\sum_{j=1 \ldots N} l^{j}(\theta) Y_{j}+A\right)\right)
$$

where the data $\left(\Gamma_{d}, l^{j}\right)$ in the formula above is defined as follows :

(i) The functions $l^{j}: \mathbb{R}^{r} \rightarrow \mathbb{R}$ are linear forms with rational coefficients ;

(ii) There exists an $r$-dimensional rational polytope $\mathcal{L} \subset \mathbb{R}^{r}$ such that

(ii.1) We have $0 \leq l^{j}(\theta) \leq 1-\varepsilon_{0}$ for all $\theta \in \mathcal{L}$, where $\varepsilon_{0}$ is a positive real.

(ii.2) The bundle $K_{X}+\sum_{j=1 \ldots N} l^{j}(\theta) Y_{j}+A \in \operatorname{Psef}(X)$ for all $\theta \in \mathcal{L}$.

(iii) The multi-index $d=\left(d^{0}, \ldots, d^{r}\right) \in \mathbb{Z}^{r+1}$, where each $d^{j}$ is divisible enough ;

(i4) A couple $(m, \theta) \in \Gamma_{d}$ if and only if $\theta \in \mathcal{L} \cap \mathbb{Q}^{r}$ and moreover $m \theta^{k} \in d_{k} \mathbb{Z}$, for $k=0,1, \ldots, r$ (by convention, we put $\theta^{0}:=1$ ).

We remark that a decomposition of the polytope $\mathcal{L}$ into simplexes induce a decomposition of the algebra $\mathcal{A}_{r}(X)$ as a direct sum, so we can assume that $\mathcal{L}$ is a $r$-dimensional simplex, generated by the vertices $\xi_{1}, \ldots, \xi_{r+1}$; by hypothesis, the coordinates $\left(\xi_{j}^{i}\right)$ of each $\xi_{j}$ is a rational number.

For each point $\theta \in \mathcal{L}$ we can define its affine coordinates

$$
\theta=\sum_{j=1}^{r+1} \eta^{j} \xi_{j}
$$

where $\eta^{j} \geq 0$ and $\sum_{j} \eta^{j}=1$. The relation (59) above implies that each $\eta^{j}$ is an affine function of the coordinates of $\theta$ (with respect to the canonical basis of $\mathbb{R}^{r}$ ).

The $\mathbb{Q}$-bundle $K_{X}+\sum_{j=1}^{N} l^{j}\left(\xi_{k}\right) Y_{j}+A$ is pseudoeffective for each $k=1, \ldots, r+1$, thanks to the property (ii). By the non-vanishing theorem [2], [22] there exists an 
effective $\mathbb{Q}$-divisor

$$
\Xi_{k}:=\sum_{j=1}^{N} a_{k}^{j} Y_{j}
$$

linearly equivalent with $K_{X}+\sum_{j=1}^{N} l^{j}\left(\xi_{k}\right) Y_{j}+A$ (strictly speaking, this is not entirely correct, since the support of the zero set of the section $\Xi_{k}$ is not obliged to be contained in $\left(Y_{j}\right)$, but it becomes so after a blow-up ; we do not detail this step here, since it is completely standard).

We notice that we can assume that $\Xi_{k} \neq 0$ for all $k=1, \ldots, r+1$. Indeed, assume that say $\Xi_{r+1}=0$; then we can reduce the study of the algebra $\mathcal{A}_{r}(X)$ to a smaller rank algebra, as follows (see [2]).

Let $\alpha \in \mathcal{L}$; we can write it as a convex combination

$$
\alpha=\left(1-\eta^{r+1}\right) \alpha_{1}+\eta^{r+1} \xi_{r+1}
$$

where $\theta_{1}$ belongs to the polytope whose vertices are $\xi_{1}, \ldots, \xi_{r}$. Since $\Xi_{r+1}=0$, we infer that the bundle $K_{X}+\sum_{j=1}^{N} l^{j}\left(\xi_{r+1}\right) Y_{j}+A$ is linearly equivalent to zero, and then the current

$$
T:=\frac{1}{1-\eta^{r+1}} \Theta_{\min }(\alpha)
$$

belongs to the class $\left\{K_{X}+\sum_{j=1}^{N} l^{j}\left(\alpha_{1}\right) Y_{j}+A\right\}$ (we denote by $\Theta_{\min }(\alpha)$ the current with minimal singularities of the class $\left.\left\{K_{X}+\sum_{j=1}^{N} l^{j}(\alpha) Y_{j}+A\right\}\right)$. We clearly have the equality

$$
\Theta_{\min }(\alpha)=\left(1-\eta^{r+1}\right) T
$$

which in turn implies that

$$
\Theta_{\min }(\alpha)=\left(1-\eta^{r+1}\right) \Theta_{\min }\left(\alpha_{1}\right)
$$

so the finite generation of the algebra $\mathcal{A}_{r}(X)$ is obtained by induction on $r$, under the assumption above (we do no treat the case $r=1$ separately, since it is completely similar to the general case).

Hence we will assume for the rest of our arguments that $\Xi_{k} \neq 0$ for all $k=1, \ldots, r+1$, and let $m_{0}$ be a positive integer, multiple of the denominators of $a_{k}^{j}, \xi_{j}^{i}$ and of the coefficients of $\left(l^{j}\right)$. We obtain a non-zero section

$$
s_{k} \in H^{0}\left(X, m_{0}\left(K_{X}+\sum_{j=1}^{N} l^{j}\left(\xi_{k}\right) Y_{j}+A\right)\right)
$$

whose zeroes divisor is $m_{0} \Xi_{k}$.

Our approach for the finite generation statement 0.1 (ii) is based upon the next simple result. 
3.1 Proposition. Let $\delta>0$ be a positive real number. We assume that there exists an integer $d_{\infty} \in \mathbb{Z}_{+}$divisible enough, and a finite set of sections

$$
\sigma_{k} \in H^{0}\left(X, m_{k}\left(K_{X}+\sum_{j=1}^{N} l^{j}\left(\theta_{k}\right) Y_{j}+A\right)\right)
$$

where $k=1, \ldots, K$ and $\left(m_{k}, \theta_{k}\right) \in \Gamma_{d}$ such that the next requirements are satisfied.

(R.1) There exists a finite set of points $\alpha_{q} \in \mathcal{L}$, together with the positive reals $\delta>$ $\delta_{q}>0$ such that $\mathcal{L} \subset \cup_{q} B\left(\alpha_{q}, \delta_{q}\right)$, where $B\left(\alpha_{q}, \delta_{q}\right)$ is the ball in $\mathbb{R}^{r}$ centered at $\alpha_{q}$ with radius $\delta_{s}$ with respect to the norm $\|\theta\|:=\sup _{j}\left|\theta^{j}\right|$.

(R.2) Let $u$ be a section of the bundle $m\left(K_{X}+\sum_{j=1, . ., N} l^{j}(\theta) Y_{j}+A\right)$ such that $(m, \theta) \in$ $\Gamma_{d}$ and moreover $m \theta^{j} \in d_{\infty} d^{j} \mathbb{Z}_{+}$for all $j=0, \ldots, r$; we assume that $\theta \in$ $B\left(\alpha_{s}, \delta_{s}\right)$. Then we have

$$
u=u_{1}+\sum_{p \in I(m, \theta)} \lambda_{d} \sigma_{1}^{\otimes p_{1}} \otimes \ldots \otimes \sigma_{K}^{\otimes p_{k}}
$$

where $I(m, \theta)$ is the set of multi-indexes $p$ such that

$$
\sum m_{k} p_{k}=m, \quad \sum p_{k} m_{k} \theta_{k}=m \theta
$$

and the section $u_{1}$ satisfies the vanishing conditions $\operatorname{ord}_{Y_{q}}\left(u_{1}\right) \geq m \delta_{s}$, for any integer $1 \leq q \leq N$ such that $\sum_{m=1}^{r+1} \eta^{m}\left(\alpha_{s}\right) a_{m}^{q} \neq 0$.

Then the algebra $\mathcal{A}_{r}(X)$ is finitely generated.

Proof. We denote by $\mathcal{A}_{r}^{\left(d_{\infty}\right)}(X)$ the truncation of $\mathcal{A}_{r}(X)$ corresponding to the degrees $m$ which satisfy the arithmetic conditions in $\mathbf{R}_{\mathbf{2}}$. A first observation is that the integer $m_{0}$ in (61) can be assumed to be a multiple of $d_{\infty}$. We will prove next that $\mathcal{A}_{r}(X)$ is finitely generated by the elements $\left(\sigma_{j}\right),\left(s_{k}\right)$, together with a finite number of pluricanonical sections of small degree.

To this end, let $u$ be a section of $m\left(K_{X}+\sum_{j=1}^{N} l^{j}(\theta) Y_{j}+A\right)$, such that $\left\|\theta-\alpha_{1}\right\| \leq \delta_{1}$. By hypothesis we have $u=P\left(\sigma_{1}, \ldots, \sigma_{N}\right)+u_{1}$ where $u_{1}$ has the vanishing properties stated in R.2. We can assume that

$$
m \delta_{1} \geq \max _{j, p}\left\{m_{0} a_{p}^{j}\right\}
$$

since otherwise $u$ belongs to a fixed, finite subset of $\mathcal{A}_{r}^{\left(d_{\infty}\right)}(X)$.

Let $k$ be an integer such that $\eta^{k}\left(\alpha_{1}\right) \neq 0$. According to the hypothesis of the proposition, we have $\operatorname{ord}_{Y_{q}}\left(u_{1}\right) \geq \delta_{1} m$ in particular for all the indexes $q$ such that $Y_{q}$ belongs to the support of $\Xi_{k}$. Then the section $u_{1}$ can be written as

$$
u_{1}=s_{k} v_{k}
$$


and we verify next that there exists some $k$ for which the section $v_{k}$ above belongs to the truncation of the algebra $\mathcal{A}_{r}(X)$ as fixed in the preceding proposition-because we are willing to use the induction on the degree of $u$. In any case we have

$$
v_{k} \in H^{0}\left(X,\left(m-m_{0}\right)\left(K_{X}+\sum_{j=1, \ldots, N} l^{j}\left(\theta^{\prime}\right) Y_{j}+A\right)\right)
$$

where $\theta^{\prime}$ is defined by the relation

$$
m_{0} \xi_{k}+\left(m-m_{0}\right) \theta^{\prime}=m \theta
$$

Our goal is to show that for some index $k$ the point $\theta^{\prime}$ belongs to the polytope $\mathcal{L}$. We remark that the affine coordinates of $\theta^{\prime}$ are given by

$$
\eta^{j}\left(\theta^{\prime}\right)=\frac{m}{m-m_{0}} \eta^{j}(\theta)
$$

if $j \neq k$ and

$$
\eta^{k}\left(\theta^{\prime}\right)=\frac{m \eta^{k}(\theta)-m_{0}}{m-m_{0}}
$$

so we will have $\theta^{\prime} \in \mathcal{L}$ if

$$
m \eta^{k}(\theta) \geq m_{0}
$$

If the inequality (64) fails to hold for all $k$ such that $\eta^{k}\left(\alpha_{1}\right) \neq 0$, then we obtain an upper bound for $m$, because $\left\|\theta-\alpha_{1}\right\| \leq \delta_{1}$, so we will have

$$
m \leq C \delta_{1} m+d m_{0}
$$

and of course we can assume that $C \delta_{1}<1$. Therefore, unless $m$ is smaller than a fixed constant, there exists an index $k$ for which the inequality (64) is verified ; moreover, the relations $\left(m-m_{0}\right) \theta^{\prime j} \in d_{\infty} d^{j} \mathbb{Z}$ follows from (63).

In conclusion, the section $v_{k}$ belongs to $\mathcal{A}_{r}^{\left(d_{\infty}\right)}(X)$, and the finite generation of this algebra is deduced by induction on the degree $m$. Thus, the proposition is completely proved, since the $d_{\infty}$ power of any section of $\mathcal{A}_{r}(X)$ belongs to $\mathcal{A}_{r}^{\left(d_{\infty}\right)}(X)$.

\section{§3.A The first step}

The construction of the generators $\left(\sigma_{j}\right)$ will be performed by induction on $\operatorname{dim}(X)$, by using the compactness of $\mathcal{L}$; we begin by fixing an arbitrary point $\alpha_{0} \in \mathcal{L}$.

By a suitable convex combination of the set of effective $\mathbb{Q}$-divisors $\Xi_{k}$ corresponding to the vertices of $\mathcal{L}$ we obtain

$$
K_{X}+\sum_{j=1}^{N} l^{j}\left(\alpha_{0}\right) Y_{j}+A \equiv \sum_{i=1}^{N} r^{i}\left(\alpha_{0}\right) Y_{i}
$$


where $r^{i}(\theta):=\sum_{p=1}^{r+1} \eta(\theta) a_{p}^{i} \geq 0$ is an affine form with rational coefficients, for each $i$, cf. (59), (60).

Let $t$ be a real number ; we consider the following identity, taken from [19] (which is nothing but the threshold of the $\mathbb{Q}$-divisor constructed in $(65)$ with respect to the measure $\frac{d \lambda}{\prod\left|\sigma_{Y_{j}}\right|^{l j}\left(\alpha_{0}\right)}$ in the language used in [22]) :

$$
(1+t)\left(K_{X}+\sum_{j=1}^{N} l^{j}\left(\alpha_{0}\right) Y_{j}+A\right) \equiv K_{X}+\sum_{j=1}^{N}\left(l^{j}\left(\alpha_{0}\right)+t r^{j}\left(\alpha_{0}\right) Y_{j}+A .\right.
$$

The procedure we will describe next can be used under more general hypothesis on the linear forms $\left(r^{j}, l^{j}\right)$; the precise requirements are the following.

Initial data. There exists $t^{0}, \delta^{0} \in \mathbb{R}_{+}$such that we have

$$
r^{j}(\theta) \geq 0, \quad l^{j}(\theta)+\operatorname{tr}^{j}(\theta) \geq 0
$$

if $\left\|\theta-\alpha_{0}\right\| \leq \delta^{0}$ and $t \geq t^{0}$. Moreover we have $l^{j}\left(\alpha_{0}\right)+t^{0} r^{j}\left(\alpha_{0}\right)<1$ for all $j=1, \ldots, N$, and $r^{j}\left(\alpha_{0}\right)>0$ for each $j \in \Lambda\left(\alpha_{0}\right)$.

We consider the set $\Lambda\left(\alpha_{0}\right):=\left\{j \in J: r^{j}\left(\alpha_{0}\right) \neq 0\right\}$; thanks to the ampleness of $A$ we can assume that the following quantities

$$
\widetilde{t^{j}}:=\frac{1-l^{j}\left(\alpha_{0}\right)}{r^{j}\left(\alpha_{0}\right)}
$$

are distinct, for $j \in \Lambda\left(\alpha_{0}\right)$.

The smallest among the real numbers above is assumed to be $\widetilde{t^{1}}$; therefore there exists $\varepsilon_{1}>0$ such that

$$
l^{j}\left(\alpha_{0}\right)+\widetilde{t^{1}} r^{j}\left(\alpha_{0}\right) \leq 1-\varepsilon_{1}
$$

for all $j \neq 1$; we also have

$$
l^{1}\left(\alpha_{0}\right)+\widetilde{t^{1}} r^{1}\left(\alpha_{0}\right)=1
$$

so we can write

$$
\left(1+\widetilde{t^{1}}\right)\left(K_{X}+\sum_{j=1}^{N} l^{j}\left(\alpha_{0}\right) Y_{j}+A\right) \equiv K_{X}+Y_{1}+\sum_{j=2}^{N}\left(l^{j}\left(\alpha_{0}\right)+\widetilde{t^{1}} r^{j}\left(\alpha_{0}\right) Y_{j}+A .\right.
$$

We have $t^{0}<\widetilde{t}^{1}$, and it may happen that $\widetilde{t^{1}}$ is not rational, so during the next lines we will construct a rational approximation $t^{1}$ of $\widetilde{t^{1}}$, and we will determine a real $\delta>0$ such that

$$
\left(1+t^{1}\right)\left(K_{X}+\sum_{j=1}^{N} l^{j}(\theta) Y_{j}+A\right) \equiv K_{X}+Y_{1}+\sum_{j=2}^{N_{1}} \widetilde{l^{j}}(\theta) Y_{j}+A_{1},
$$


where $\widetilde{l^{j}}$ are linear forms with rational coefficients, such that $0 \leq \widetilde{l^{j}}(\theta) \leq 1-\frac{\varepsilon_{1}}{2}$ if $\left\|\theta-\alpha_{0}\right\| \leq \delta$.

In order to achieve this, we will use the same arguments as in the paragraph 2.B. Let $\gamma, \delta$ be positive real numbers and let $t^{1} \in\left[(1-\gamma) \widetilde{t^{1}}, \widetilde{t^{1}}\right] \cap \mathbb{Q}$. For any $\theta \in \mathcal{L}$ such that $\left\|\theta-\alpha_{0}\right\| \leq \delta$, a quick computation shows that we have

$$
\left|l^{j}\left(\alpha_{0}\right)+\widetilde{t^{1}} r^{j}\left(\alpha_{0}\right)-l^{j}(\theta)-t^{1} r^{j}(\theta)\right| \leq C\left(\widetilde{t^{1}}\right)(\gamma+\delta)
$$

where $C\left(\widetilde{t^{1}}\right) \geq 1$ is a constant depending on $\widetilde{t^{1}}$. The first requirement we impose to $\delta$ and $\gamma$ is

$$
C\left(\widetilde{t^{1}}\right)(\gamma+\delta) \leq \frac{\varepsilon_{1}}{2}
$$

and then we will have

$$
0 \leq l^{j}(\theta)+t^{1} r^{j}(\theta) \leq 1-\frac{\varepsilon_{1}}{2}
$$

if $\left\|\theta-\alpha_{0}\right\| \leq \delta$ and $j \neq 1$.

The next conditions on $\delta, \gamma$ are needed in order to transform the expression $\left(l^{1}(\theta)+\right.$ $\left.t^{1} r^{1}(\theta)-1\right) Y_{1}+A$. Let

$$
l_{0}:=\max _{j} l^{j}\left(\alpha_{0}\right)<1
$$

and let $C_{A}$ be a positive and divisible enough integer, such that

$$
C_{A}\left(1-l_{0}\right) \geq 2
$$

and such that $C_{A} A+Y_{j}, C_{A} A-Y_{j}$ and $C_{A} A$ are very ample line bundles. Let $q \geq$ $C\left(\widetilde{t^{1}}\right)(\gamma+\delta)$ be a rational number ; we have

$$
\left(l^{1}(\theta)+t^{1} r^{1}(\theta)-1\right) Y_{1}+A \equiv \sum_{j=1}^{3} \widetilde{l}^{N+j}(\theta) H_{j}+1 / 2 A
$$

where $H_{N+1}, H_{N+2}, H_{N+3}$ are respectively generic hyperplane sections of the linear systems $\left|Y_{1}+C_{A} A\right|,\left|C_{A} A-Y_{1}\right|$ and $\left|C_{A} A\right|$, and where we define

$$
\widetilde{l}^{N+1}(\theta):=l^{1}(\theta)+t^{1} r^{1}(\theta)-1+q, \quad \widetilde{l}^{N+2}(\theta):=q,
$$

and

$$
\widetilde{l}^{N+3}(\theta):=\left(1+\frac{1}{2 C_{A}}-l^{1}(\theta)-t^{1} r^{1}(\theta)-2 q\right)
$$

We choose now the parameters as follows

$$
\gamma^{1}:=\min \left(\frac{\varepsilon_{1}}{4 C\left(\widetilde{t^{1}}\right)}, \frac{1}{12 C_{A} C\left(\widetilde{t^{1}}\right)}, \frac{2 C_{A}-1}{2 C_{A}\left(1-\min _{i} l^{i}\left(\alpha_{0}\right)\right)}\right) \leq \frac{1}{2},
$$

$q:=\varepsilon_{1}$ and let $t^{1}$ be any rational number contained in the interval $\left[\left(1-\gamma^{1}\right) \widetilde{t^{1}}, \widetilde{t^{1}}\right]$; we do not yet fix $\delta$, but instead impose the condition $\delta \leq \gamma^{1}$. The first two quantities in (73) are dictated by (70) and the bounds we wish to impose to the affine forms $\widetilde{l}^{N+j}$. 
In conclusion, we have the linear equivalence relation

$$
\left(1+t^{1}\right)\left(K_{X}+\sum_{j=1}^{N} l^{j}(\theta) Y_{j}+A\right) \equiv K_{X}+Y_{1}+\sum_{j=2}^{N+3} \widetilde{l^{j}}(\theta) Y_{j}+A_{1},
$$

where $A_{1}=1 / 2 A$, and $\widetilde{l^{j}}(\theta):=l^{j}(\theta)+t^{1} r^{j}(\theta)$ for $j=2, \ldots, N$.

As a motivation for what will follow, we recall that in order to be able to apply the proposition 3.1 above, we have to show in particular that the algebra $\mathcal{A}_{r}(X)$ is finitely generated modulo the sections whose normalized vanishing order along $Y_{1}$ is greater than a fixed constant. Thus, it is natural to consider the expression

$$
\begin{aligned}
\left(1+t^{1}\right)\left(K_{X}+\sum_{j=1}^{N} l^{j}(\theta) Y_{j}+A\right)-\theta^{r+1} Y_{1} & \equiv K_{X}+Y_{1}+\sum_{j=2}^{N+3} \widetilde{l^{j}}(\theta) Y_{j}+ \\
& +A_{1}-\theta^{r+1} Y_{1}
\end{aligned}
$$

where the additional parameter $\theta^{r+1}$ corresponds to the normalized vanishing order along $Y_{1}$. The last part in the formula (75) can be written as follows

$$
A_{1}-\theta^{r+1} Y_{1}=\theta^{r+1}\left(2 C_{A} A_{1}-Y_{1}\right)+\left(1 / 2-2 C_{A} \theta^{r+1}\right) A_{1}+1 / 2 A_{1} .
$$

Next we define $\varepsilon^{1}$ by the next equality

$$
\left(1+t^{1}\right) \varepsilon^{1}:=\frac{1}{4 C_{A}}
$$

and then we have

$$
\left(1+t^{1}\right)\left(K_{X}+\sum_{j=1}^{N} l^{j}(\theta) Y_{j}+A\right)-\theta^{r+1} Y_{1} \equiv K_{X}+Y_{1}+\sum_{j=2}^{N+5} \widetilde{l^{j}}\left(\theta, \theta^{r+1}\right) Y_{j}+A_{2}
$$

where the forms/hypersurfaces corresponding to $j=N+4, N+5$ are obtained via (76), and $A_{2}=1 / 4 A$. The choice of $\varepsilon^{1}$ as in (77), together with (73) and the fact that $t^{1} \geq\left(1-\gamma^{1}\right) \widetilde{t}^{1}$ implies

$$
r^{1}\left(\alpha_{0}\right) \geq 2 \varepsilon^{1}, \quad l^{1}\left(\alpha_{0}\right)+t^{1} r^{1}\left(\alpha_{0}\right) \geq 2\left(1+t^{1}\right) \varepsilon^{1} .
$$

Now we fix $\delta^{1} \leq \gamma^{1}$ small enough in order to insure that

$$
r^{1}(\theta) \geq \varepsilon^{1}, \quad l^{1}(\theta)+t^{1} r^{1}(\theta) \geq\left(1+t^{1}\right) \varepsilon^{1}
$$

for any $\theta \in \mathcal{L}$ such that $\left\|\theta-\alpha_{0}\right\| \leq \delta^{1}$. This implies that

$$
l^{1}(\theta)+t r^{1}(\theta) \geq(1+t) \varepsilon^{1} .
$$

for any $t \geq t^{1}$. If $0 \leq \theta^{r+1} \leq\left(1+t^{1}\right) \varepsilon^{1}$ and $\left\|\theta-\alpha_{0}\right\| \leq \delta^{1}$ we notice that $0 \leq$ $\tilde{l^{j}}\left(\theta, \theta^{r+1}\right) \leq 1-\varepsilon_{1} / 2$. 
After all this preliminaries, we introduce the following algebra. We first define the polytope $\mathcal{L}^{\prime} \subset \mathcal{L} \times\left[0, \varepsilon^{1}\left(1+t^{1}\right)\right]$ given by the couples $\left(\theta, \theta^{r+1}\right)$ satisfying the following conditions :

(a) We have $\left\|\theta-\alpha_{0}\right\| \leq \delta^{1}$;

(b) The bundle $K_{X}+Y_{1}+\sum_{j=2}^{N+5} \tilde{l^{j}}\left(\theta, \theta^{r+1}\right) Y_{j}+A_{2} \in \operatorname{Psef}(X)$, and its generic Lelong number across $Y_{1}$ is equal to zero.

The fact that indeed $\mathcal{L}^{\prime}$ is a polytope is a consequence of the corollary 2.C.7.

Now we recall that in the definition of the algebra $\mathcal{A}_{r}(X)$ we use the element $d=\left(d^{0}, \ldots, d^{r}\right) \in \mathbb{Z}^{r+1}$ in order to define the set $\Gamma_{d}$. Let $n_{1}$ be an integer such that $\frac{n_{1}}{1+t^{1}} \in \mathbb{Z} ;$ we introduce the following set

$$
\Gamma_{d}^{\prime}:=\left\{\left(m^{\prime}, \theta^{\prime}\right): \theta^{\prime}=\left(\theta, \theta^{r+1}\right) \in \mathcal{L}^{\prime} \cap \mathbb{Q}^{r+1}, \quad m^{\prime} \theta^{j} \in \frac{n_{1}}{1+t^{1}} d^{j} \mathbb{Z}_{+}, \quad m^{\prime} \theta^{r+1} \in \mathbb{Z}\right\}
$$

and the associated algebra

$$
\mathcal{A}_{r+1}\left(X, \alpha_{0}\right):=\bigoplus_{\left(m^{\prime}, \theta^{\prime}\right) \in \Gamma_{d}^{\prime}} H^{0}\left(X, m^{\prime}\left(K_{X}+Y_{1}+\sum_{j=1}^{N+5} \widetilde{l^{j}}\left(\theta^{\prime}\right) Y_{j}+A_{2}\right)\right) .
$$

We remark that the previous definition is meaningful, as soon as $n_{1}$ is divisible enough. We also remark the asymmetry of the arithmetic conditions imposed to $\theta$ and $\theta^{r+1}$ in the definition of $\Gamma_{d}^{\prime}$; the reasons for this are easily guessed from the formulas (77)-(78), and they will appear even more clearly in a moment.

Let $\mathcal{A}_{r+1}\left(X, \alpha_{0}\right)_{\mid Y_{1}}$ be the restricted algebra associated to $\mathcal{A}_{r+1}\left(X, \alpha_{0}\right)$; we have the next simple consequence of 2.C.7.

3.A.1 Lemma. The restricted algebra $\mathcal{A}_{r+1}\left(X, \alpha_{0}\right)_{\mid Y_{1}}$ is finitely generated.

Proof. Our first observation is that the coefficients of $Y_{j}$ above verify the inequality $0 \leq \widetilde{l^{j}}\left(\theta^{\prime}\right) \leq 1-\epsilon_{1} / 2$, for each $\theta^{\prime} \in \mathcal{L}^{\prime}$, as a consequence of the definitions/choices previously made. In this context, there exists a birational map $\mu: \widehat{X} \rightarrow X$ such that -up to a subdivision of $\mathcal{L}^{\prime}$ - we have

$\left.\mu^{\star}\left(K_{X}+Y_{1}+\sum_{j=1}^{N+5} \widetilde{l^{j}}\left(\theta^{\prime}\right) Y_{j}+A_{2}\right)\right)+\sum_{i \in I_{-}} \widehat{l^{i}}\left(\theta^{\prime}\right) E_{i}=K_{\widehat{X}}+\widehat{Y_{1}}+\sum_{i \in I_{+}} \widehat{l^{j}}\left(\theta^{\prime}\right) \widehat{Y_{j}}+\mu^{\star}\left(A_{2}\right)$

where the hypersurfaces $\widehat{Y_{j}}$ corresponding to $I_{+}$are mutually disjoint, and the $\mu$-proper transform of $Y_{1}$ is equal to its total inverse image (again, we refer to the arguments in [14] for the existence of $\mu$ ).

As it was already discussed in the first paragraph of the present article (and also [1], [14]), via the map $\mu$ we can easily identify a truncation of the restricted algebra $\mathcal{A}_{r+1}\left(X, \alpha_{0}\right)_{\mid Y_{1}}$, as follows.

For each $\theta^{\prime} \in \mathcal{L}^{\prime}$, the current with minimal singularities corresponding to the bundle $K_{X}+Y_{1}+\sum_{j=2}^{N+5} \widetilde{l^{j}}\left(\theta^{\prime}\right) Y_{j}+A_{2}$ admits a well-defined restriction to $Y_{1}$, and let $\rho_{\min }^{j}\left(\theta^{\prime}\right)$ 
be the Lelong number of this restriction at the generic point of $Y_{j \mid Y_{1}}$. By the corollary 2.C.7, the functions $\rho_{\min }^{j}$ are piecewise affine, defined over $\mathbb{Q}$, and let $p_{0}$ be an integer, which is a multiple of all the denominators of their coefficients. Then the $p_{0}$-truncation of the restricted algebra $\mathcal{A}_{r+1}\left(X, \alpha_{0}\right)_{\mid Y_{1}}$ is isomorphic to

$$
\mathcal{A}:=\bigoplus_{\left(m^{\prime}, \theta^{\prime}\right) \in \Gamma_{p_{0} d}^{\prime}} H^{0}\left(\widehat{Y_{1}}, m^{\prime}\left(K_{\widehat{Y}_{1}}+\sum_{j \in I_{+}}\left(\widetilde{l j}\left(\theta^{\prime}\right)-\rho_{\min }^{j}\left(\theta^{\prime}\right)\right)_{+} \widehat{Y}_{j \mid \widehat{Y}_{1}}+\mu^{\star}\left(A_{2}\right)\right)\right) \text {. }
$$

where the set $\Gamma_{p_{0} d}^{\prime}$ in the expression above is defined as $\left(m^{\prime}, \theta^{\prime}\right) \in \Gamma_{d}^{\prime}$ such that

$$
m^{\prime} \theta^{k} \in p_{0} d^{k} \frac{n_{1}}{1+t^{1}} \mathbb{Z}_{+}
$$

if $0 \leq k \leq r$ and $m^{\prime} \theta^{r+1} \in p_{0} \mathbb{Z}$. By induction, the algebra $\mathcal{A}$ is finitely generated, and then the restricted algebra has the same property ; thus the lemma 3.A.1 is completely proved.

3.A.2 Remark. By the construction of the algebra $\mathcal{A}_{r+1}\left(X, \alpha_{0}\right)$ we infer the following fact, which will play an important role for the rest of the proof of 0.1 . Let

$$
v \in H^{0}\left(X, m^{\prime}\left(K_{X}+Y_{1}+\sum_{j=1}^{N+5} \widetilde{l^{j}}\left(\theta^{\prime}\right) Y_{j}+A_{2}\right)\right)
$$

be an element of this algebra. We have $\theta^{\prime}=\left(\theta, \theta^{r+1}\right)$, and we consider the section

$$
u:=v \otimes \sigma_{Y_{1}}^{\otimes m^{\prime} \theta^{r+1}}
$$

we denote by $\sigma_{Y_{1}}$ the canonical section associated to $Y_{1}$. The above definition is legitimate, since by the definition of $\Gamma_{d}^{\prime}$ we have $m^{\prime} \theta^{r+1} \in \mathbb{Z}_{+}$.

The claim is that we have

$$
u \in A_{r}(X)
$$

and indeed this is obvious, since by the relation (78) we have

$$
u \in H^{0}\left(X, m^{\prime}\left(1+t^{1}\right)\left(K_{X}+\sum_{j=1}^{N} l^{j}(\theta) Y_{j}+A\right)\right)
$$

as well as $\theta \in \mathcal{L}$ and $m^{\prime}\left(1+t^{1}\right) \theta^{j} \in n_{1} d^{j} \mathbb{Z}_{+}$, for $j=0, \ldots, r$.

Before explaining the end of our proof, we will discuss next the impact of the finite generation of the restricted algebra $\mathcal{A}_{r+1}\left(X, \alpha_{0}\right)_{\mid Y_{1}}$ on the properties of our initial object $\mathcal{A}_{r}(X)$.

Let $\mathcal{A}_{r}^{\left(n_{1}\right)}(X)$ be the truncation of $\mathcal{A}_{r}(X)$, corresponding to the set $(m, \theta) \in \Gamma_{d}$ such that $m \theta^{k} \in n_{1} d^{k} \mathbb{Z}$, for $k=0, \ldots, r$ (where the integer $n_{1}$ is the same as the one appearing in the construction of the algebra $\mathcal{A}_{r+1}\left(X, \alpha_{0}\right)$ a few lines above). Let

$$
u \in H^{0}\left(X, m\left(K_{X}+\sum_{j=1}^{N} l^{j}(\theta) Y_{j}+A\right)\right)
$$


be an element of $\mathcal{A}_{r}^{\left(n_{1}\right)}(X)$, such that $\left\|\theta-\alpha_{0}\right\| \leq \delta$. We write $m=\left(1+t^{1}\right) m^{\prime}$ where $m^{\prime} \in \mathbb{Z}$ thanks to the fact that $m \in\left(1+t^{1}\right) \mathbb{Z}$. By the formula (74), the section $u$ becomes an element of the group

$$
H^{0}\left(X, m^{\prime}\left(K_{X}+Y_{1}+\sum_{j=2}^{N+3} \widetilde{l^{j}}(\theta) Y_{j}+A_{1}\right)\right)
$$

(because we tacitly assume that $n_{1}$ is divisible enough). Let $k_{1}$ be the vanishing order of $u$ along the hypersurface $Y_{1}$; we have

$$
u=\sigma_{Y_{1}}^{\otimes k_{1}} u_{1}
$$

If $\frac{k_{1}}{m} \geq \varepsilon^{1}$, then we stop ; if not, we observe that $u_{1}$ is a section of the bundle

$$
m^{\prime}\left(K_{X}+Y_{1}+\sum_{j=1}^{N+5} \widetilde{l^{j}}\left(\theta^{\prime}\right) Y_{j}+A_{2}\right)
$$

where $\theta^{r+1}=k / m^{\prime}$. In other words, $u_{1}$ is an element of $\mathcal{A}_{r+1}\left(X, \alpha_{0}\right)$, as one can deduce from the relations (a), (b) and the definition of $\Gamma_{d}^{\prime}$.

We denote by $\beta_{1}, \ldots, \beta_{K}$ the generators of the restricted algebra $\mathcal{A}_{r+1}\left(X, \alpha_{0}\right)_{\mid Y_{1}}$, and then we have

$$
u_{1}=\sum_{p} \lambda_{p} \beta_{1}^{\otimes p_{1}} \otimes \ldots \otimes \beta_{K}^{\otimes p_{K}}+\sigma_{Y_{1}}^{k_{2}} u_{2}
$$

where $k_{2} \geq 1$ and where the restriction of $u_{2}$ to $Y_{1}$ is non-identically zero. that

If $\beta_{k} \in H^{0}\left(X, m_{k}^{\prime}\left(K_{X}+Y_{1}+\sum_{j=1}^{N+5} \widetilde{l^{j}}\left(\theta_{k}^{\prime}\right) Y_{j}+A_{2}\right)\right)$, then the equality above implies

$$
m^{\prime}\left(\theta, \theta^{r+1}\right)=\sum_{k=1}^{K} p_{k} m_{k}^{\prime}\left(\theta_{k}, \theta_{k}^{r+1}\right)
$$

so we have

$$
m^{\prime} \theta^{r+1}=\sum_{k=1}^{K} p_{k} m_{k}^{\prime} \theta_{k}^{r+1}
$$

For $k=1, \ldots, K$, we define

$$
\omega_{k}:=\beta_{k} \otimes \sigma_{Y_{1}}^{m_{k}^{\prime} \theta_{k}^{r+1}} \in H^{0}\left(X,\left(1+t^{1}\right) m_{k}^{\prime}\left(K_{X}+\sum_{j=1}^{N} l^{j}\left(\theta_{k}\right) Y_{j}+A\right)\right) .
$$

Thanks to the remark 3.A.2 above, we have $\omega_{k} \in \mathcal{A}_{r}^{\left(n_{1}\right)}(X)$ and we get

$$
u=\sum_{s} \lambda_{p} \omega_{1}^{\otimes p_{1}} \otimes \ldots \otimes \omega_{K}^{\otimes p_{K}}+\sigma_{Y_{1}}^{k_{1}+k_{2}} u_{2}
$$

Again, if $\frac{k_{1}+k_{2}}{m} \geq \varepsilon^{1}$, then we stop ; if not, we repeat the same procedure with $u_{2}$. 
In conclusion, after a finite number of steps, we can write

$$
u=\sum_{s} \lambda_{s} \omega_{1}^{\otimes s_{1}} \otimes \ldots \otimes \omega_{K}^{\otimes s_{K}}+\sigma_{Y_{1}}^{\otimes m \varepsilon^{1}} u_{[2]}
$$

where $u_{[2]}$ is a section of the bundle $m\left(K_{X}+\sum_{j=1}^{N} l^{j}(\theta) Y_{j}+A\right)-m \varepsilon^{1} Y_{1}$.

Therefore, after this first step we have shown that our algebra verifies a consistent part of the requirements of the proposition 3.1.

\section{$\S 3 . B$ Iteration scheme}

We turn now our attention to the sections of the algebra $\mathcal{A}_{r}(X)$, whose normalized vanishing order along $Y_{1}$ is greater than $\varepsilon^{1}$.

Let $t \geq t^{1}$; we have the identity

$$
\begin{aligned}
(1+t)\left(K_{X}+\sum_{j=1}^{N} l^{j}(\theta) Y_{j}+A-\varepsilon^{1} Y_{1}\right) & \equiv K_{X}+\left(l^{1}(\theta)+t r^{1}(\theta)-(1+t) \varepsilon^{1}\right) Y_{1}+ \\
& +\sum_{j \in J, j \neq 1}\left(l^{j}(\theta)+t r^{j}(\theta) Y_{j}+A\right.
\end{aligned}
$$

In order to make the exposition a bit cleaner, we introduce further notations :

$\bullet l_{2}^{j}:=l^{j}$ and $r_{2}^{j}:=r^{j}$, if $j \neq 1$, and

- $l_{2}^{1}:=l^{1}-\varepsilon^{1}$ and $r_{2}^{1}:=r^{1}-\varepsilon^{1}$ for $j=1$.

Then we observe that we have

$$
r_{2}^{j}(\theta) \geq 0, \quad l_{2}^{j}(\theta)+t r_{2}^{j}(\theta) \geq 0
$$

for any $\theta \in \mathcal{L}$ such that $\left\|\theta-\alpha_{0}\right\| \leq \delta^{1}$, and for any $t \geq t^{1}$. For $j=1$, we remark that this is a consequence of the inequality (81), whose importance appear clearly at this moment.

We also note that we have

$$
l_{2}^{j}\left(\alpha_{0}\right)+t^{1} r_{2}^{j}\left(\alpha_{0}\right)<1, \quad r_{2}^{p}\left(\alpha_{0}\right)>0
$$

for all $j=1, \ldots, N$ and $p \in \Lambda\left(\alpha_{0}\right)$. Therefore, after the first step, the new affine forms $\left(l_{2}^{j}, r_{2}^{j}\right)$ are satisfying the assumptions in the "initial data" of the preceding section.

We consider the quantities

$$
\widetilde{t_{2}^{j}}:=\frac{1-l_{2}^{j}\left(\alpha_{0}\right)}{r_{2}^{j}\left(\alpha_{0}\right)}
$$

for $j \in \Lambda\left(\alpha_{0}\right)$; we remark that the smallest of them is strictly greater than $t^{1}$, by the inequality (87). 
The procedure described in 3.A applied in the current setting will give the numbers $\gamma^{2}, \delta^{2}, \varepsilon^{2}$ and $t^{2} \geq t^{1}$ such that the next relations are verified

$$
\left(1+t^{2}\right) \varepsilon^{2}:=\frac{1}{4 C_{A}}
$$

and

$$
r_{2}^{j_{2}}(\theta) \geq \varepsilon^{2}, \quad l_{2}^{j_{2}}(\theta)+t r_{2}^{j_{2}}(\theta) \geq(1+t) \varepsilon^{2} .
$$

for any $\theta \in \mathcal{L}$ such that $\left\|\theta-\alpha_{0}\right\| \leq \delta^{2}$ and for any $t \geq t^{2}$. The integer $j_{2}$ is the index for which the minimum of the quantities (88) is achieved ; we note that we have $j_{2} \in \Lambda\left(\alpha_{0}\right)$.

We observe that we are not forced to increase the constant $C_{A}$, because the condition $C_{A}\left(1-\max _{j} l_{2}^{j}\left(\alpha_{0}\right)\right) \geq 1$ is automatically satisfied, since $l_{2}^{j} \leq l^{j}$. Also, we will get the analogue of the relation (78) as follows

$$
\left(1+t^{2}\right)\left(K_{X}+\sum_{j=1}^{N} l_{2}^{j}(\theta) Y_{j}+A\right)-\theta^{r+1} Y_{1} \equiv K_{X}+Y_{j_{2}}+\sum_{j=1, . ., N+5, j \neq j_{2}} \widetilde{l_{2}^{j}}\left(\theta, \theta^{r+1}\right) Y_{j}+A_{2} .
$$

With this data, we introduce the analogue of the algebra $\mathcal{A}_{r+1}\left(X, \alpha_{0}\right)$, and at the end of the second step, we will obtain the following conclusion. If $j_{2}=1$, then we get the finite generation of the algebra $\mathcal{A}_{r}(X)$ locally near the point (a-priori chosen) $\alpha_{0}$, modulo sections having the normalized vanishing order along $Y_{1}$ greater than $\varepsilon_{1}+\varepsilon_{2}$. If not, the same conclusion holds, modulo sections with normalized vanishing order greater than $\varepsilon_{1}$ along $Y_{1}$ and greater than $\varepsilon^{2}$ along $Y_{j_{2}}$.

The rest is clear : we keep on iterating the procedure above, so assume that we have performed this say $k$ times. This means that for each $1 \leq p \leq k$ we have constructed the following objects.

(a) We have the linear forms $l_{p}^{j}$ and $r_{p}^{j}$, such that

$$
l_{p}^{j}:=l^{j}-\sum_{q \in \Lambda_{j}, q \leq p-1} \varepsilon^{q}, \quad r_{p}^{j}:=r^{j}-\sum_{q \in \Lambda_{j}, q \leq p-1} \varepsilon^{q}
$$

where we have $q \in \Lambda_{j}$ if and only if the index $j$ achieve the minimum of the expressions (88) at the $q^{\text {th }}$ step.

(b) We obtain a set of real numbers $\left(\gamma^{p}, \delta^{p}, \varepsilon^{p}, t^{p}\right)$ such that

$$
\min _{j=1, \ldots, N} \frac{1-l_{p}^{j}\left(\alpha_{0}\right)}{r_{p}^{j}\left(\alpha_{0}\right)} \geq t^{p} \geq t^{p-1}, \quad\left(1+t^{p}\right) \varepsilon^{p}:=\frac{1}{4 C_{A}}
$$

and such that

$$
r_{p}^{j}(\theta) \geq 0, \quad l_{p}^{j}(\theta)+t r_{p}^{j}(\theta) \geq 0
$$

together with

$$
r_{p}^{q}\left(\alpha_{0}\right)>0, \quad r_{p}^{j_{p}}(\theta) \geq \varepsilon^{p}, \quad l_{p}^{j_{p}}(\theta)+t r_{p}^{j_{p}}(\theta) \geq(1+t) \varepsilon^{p}
$$


if $\left\|\theta-\alpha_{0}\right\| \leq \delta^{p}, t \geq t^{p}$ and $q \in \Lambda\left(\alpha_{0}\right)$; we denote by $j_{p}$ the index for which the minimum of the quantities (88) is achieved at the begining of the step $p$.

(c) We have the identity

$$
\begin{aligned}
& \left(1+t^{p}\right)\left(K_{X}+\sum_{j=1}^{N} l_{p}^{j}(\theta) Y_{j}+A\right)-\theta^{r+1} Y_{j_{p}} \equiv \\
& \equiv K_{X}+Y_{j_{p}}+\sum_{j=1, . ., N+5, j \neq j_{p}} \widetilde{l_{p}^{j}}\left(\theta, \theta^{r+1}\right) Y_{j}+A_{2} .
\end{aligned}
$$

(d) We define the polytope $\mathcal{L}_{p}^{\prime} \subset \mathcal{L} \times\left[0, \varepsilon^{p}\left(1+t^{p}\right)\right]$ given by the couples $\left(\theta, \theta^{r+1}\right)$ satisfying the following conditions :

$\left(d^{\prime}\right)$ We have $\left\|\theta-\alpha_{0}\right\| \leq \delta^{p} ;$

$\left(d^{\prime \prime}\right)$ The bundle $K_{X}+Y_{j_{p}}+\sum_{j \neq j_{p}} \widetilde{l_{p}^{j}}\left(\theta, \theta^{r+1}\right) Y_{j}+A_{2} \in \operatorname{Psef}(X)$, and its generic Lelong number across $Y_{j_{p}}$ is equal to zero.

(e) Let $n_{p}$ be an integer which is divisible enough, such that $\frac{n_{p}}{1+t^{p}} \in \mathbb{Z} ;$ we introduce the following set

$\Gamma_{d}^{\prime}(p):=\left\{\left(m^{\prime}, \theta^{\prime}\right): \theta^{\prime}=\left(\theta, \theta^{r+1}\right) \in \mathcal{L}_{p}^{\prime} \cap \mathbb{Q}^{r+1}, \quad m^{\prime} \theta^{j} \in \frac{n_{p}}{1+t^{p}} d^{j} \mathbb{Z}_{+}, \quad m^{\prime} \theta^{r+1} \in \mathbb{Z}\right\}$

and the associated algebra

$$
\mathcal{A}_{r+1}\left(X, \alpha_{0} ; p\right):=\bigoplus_{\left(m^{\prime}, \theta^{\prime}\right) \in \Gamma_{d}^{\prime}(p)} H^{0}\left(X, m^{\prime}\left(K_{X}+Y_{j_{p}}+\sum_{j \neq j_{p}} \widetilde{l_{p}^{j}}\left(\theta^{\prime}\right) Y_{j}+A_{2}\right)\right) .
$$

We note that the divisibility constraints we have to impose to $n_{p}$ also depends on the previous steps, as $m^{\prime} \theta^{j}$ must clear the denominators of the coefficients of of $\widetilde{l_{p}^{q}}$ for $q=1, \ldots, N+5$; in particular, we require $n_{p} \varepsilon^{q} \in \mathbb{Z}$ for each $q \leq p$. However, the total number of such constraints is finite.

As we did after the proof of the lemma 3.A.1, we explain next the progression we have achieved in the direction of the lemma 3.1 after $k \geq 2$ iterations.

In the first place, the analogue of the remark 3.A.2 reads in the iterated context as follows. Let

$$
v \in H^{0}\left(X, m^{\prime}\left(K_{X}+Y_{j_{k}}+\sum_{j \neq j_{k}} \widetilde{l_{k}^{j}}\left(\theta^{\prime}\right) Y_{j}+A_{2}\right)\right)
$$

be an element of $\mathcal{A}_{r+1}\left(X, \alpha_{0} ; k\right)$. Then we define

$$
u:=v \otimes \sigma_{Y_{j_{k}}}^{\otimes m^{\prime} \theta^{r+1}} \otimes \prod_{p=1}^{k-1} \sigma_{Y_{j_{p}}}^{\otimes m \varepsilon^{p}}
$$

where $m:=\left(1+t^{k}\right) m^{\prime}$; we remark that the above expressions are meaningful, by the divisibility properties of the $\left(n_{p}\right)$ in the point (e). The observation is that the section $u$ above belongs to the algebra $\mathcal{A}_{r}(X)$; the verification is immediate. 
Next, we define $n^{k}:=\prod_{p=1}^{k} n_{p}$, and let $\mathcal{A}_{r}^{\left(n^{k}\right)}(X)$ be the truncation of $\mathcal{A}_{r}(X)$, corresponding to the set $(m, \theta) \in \Gamma_{d}$ such that $m \theta^{s} \in n^{k} d^{s} \mathbb{Z}$, for $s=0, \ldots, r$. Let

$$
\sigma \in H^{0}\left(X, m\left(K_{X}+\sum_{j=1}^{N} l^{j}(\theta) Y_{j}+A\right)\right)
$$

be an element of $\mathcal{A}_{r}^{\left(n^{k}\right)}(X)$; we assume that $\left\|\theta-\alpha_{0}\right\| \leq \min _{j} \delta^{j}$. By the relation $(\star)$, the section $\sigma$ can be written as a polynomial of a fixed number of generators, plus $\sigma_{Y_{j_{1}}}^{\otimes m \varepsilon^{1}} \sigma_{[2]}$, where

$$
\sigma_{[2]} \in H^{0}\left(X, m\left(K_{X}+\sum_{j=1}^{N} l_{2}^{j}(\theta) Y_{j}+A\right)\right) .
$$

Let $\nu$ be the vanishing order of $\sigma_{[2]}$ along the hypersurface $Y_{j_{2}}$. If $\nu / m \geq \varepsilon^{2}$, then we stop ; if not, there exists a section

$$
v_{[2]} \in H^{0}\left(X, m^{(2)}\left(K_{X}+Y_{j_{2}}+\sum_{j \neq j_{2}} \widetilde{l_{2}^{j}}\left(\theta^{\prime}\right) Y_{j}+A_{2}\right)\right)
$$

such that

$$
\sigma_{[2]}=\sigma_{Y_{j_{2}}}^{\otimes \nu} v_{[2]}
$$

where $m=\left(1+t^{2}\right) m^{(2)}$ (thanks to the relations (93) above). In other words, the section $v_{[2]}$ becomes an element of the algebra $\mathcal{A}_{r+1}\left(X, \alpha_{0} ; 2\right)$.

The procedure described at the end of 3.A will show that the section $u$ is equivalent with $\sigma_{Y_{j_{1}}}^{\otimes m \varepsilon^{1}} \sigma_{Y_{j_{2}}}^{\otimes m \varepsilon^{2}} \sigma_{[3]}$, modulo a polynomial of a finite number of generators (corresponding to the finite generation of the restricted algebras of $\mathcal{A}_{r+1}\left(X, \alpha_{0}\right)$ and $\left.\mathcal{A}_{r+1}\left(X, \alpha_{0} ; 2\right)\right)$.

In conclusion, after $k$ steps we obtain

$$
u=\sum_{s} \lambda_{s} \omega_{1}^{\otimes s_{1}} \otimes \ldots \otimes \omega_{F}^{\otimes s_{F}}+\sigma_{Y_{j_{1}}}^{\otimes m \varepsilon^{1}} \otimes \ldots \otimes \sigma_{Y_{j_{k}}}^{\otimes m \varepsilon^{k}} \otimes u_{[k+1]}
$$

The following lemma shows that if $k \gg 0$, then the hypothesis of 3.1 will be fulfilled.

3.B.1 Lemma. After a finite number of iterations of the procedure above, each $j \in$ $\Lambda\left(\alpha_{0}\right)$ will be the index for which the minimum of the quantities (88) is obtained.

Proof. We argue by contradiction : assume that there exists an index $j_{0} \in \Lambda\left(\alpha_{0}\right)$ such that the minimum of (88) is not obtained for $j=j_{0}$, for any number of iterations. Then we infer that the sequence $\left(t^{k}\right)$ is bounded, because at each step $t^{k}$ is a minimum, in particular smaller than

$$
\frac{1-l^{j_{0}}\left(\alpha_{0}\right)}{r^{j_{0}}\left(\alpha_{0}\right)}
$$


and this quantity will be unchanged during the whole iteration process. Then the relation (92) gives a lower bound for $\varepsilon^{k}$, and this contradicts the positivity of $r_{j}^{p}$ for some index $j$, for which the cardinal of the set $\Lambda_{j}$ is large enough. Thus the lemma is proved, and so it is the theorem 0.1 .

3.B.2 Remark. Unfortunately, the arguments provided in this section in order to infer the finite generation statement depends too much on the fact that we have the strict positivity term $A$ in the boundary. This is a minor difficulty, and can be bypassed by a modified version of the statements 2.C.1, respectively 2.C.2 (which will be discussed elsewhere). However, it seems to us that the severe obstacle in order to remove the ampleness $A$ from 0.1 is the identification and analysis of the restricted algebra.

3.B.3 Remark. In the finite generation paragraph 10 of the article [19], starting from (the analogue of) $\mathcal{A}_{r}(X)$ it is proposed an ingenious construction of an enlarged algebra in order to conclude. It seems to us that the arguments presented this paper are incomplete, for the reasons we will detail next.

The enlarged algebra used (on pages 39-40 of [19]) can be expressed as

$$
\mathrm{R}_{j}:=\bigoplus_{(q, \tau, \rho) \in \mathcal{C}_{j}} H^{0}\left(X, q\left(K_{X}+F_{j}+\sum_{k \neq j}\left(\rho_{k}-f_{k}(\tau)\right) F_{k}+A\right)\right)
$$

where the set $\mathcal{C}_{j}$ is given by the following conditions :

(1) $\tau \in \Delta$, where $\Delta$ is the standard simplex in $\mathbb{R}^{N}$;

(2) For each $k \neq j$ we have $\delta_{k}(\tau) \leq \rho_{k}-f_{k}(\tau) \leq 1$, where $\delta_{k}$ and $f_{k}$ are affine functions of $\tau$ with rational coefficients ;

(3) We have $q \in \mathbb{R}_{+}$such that for each $k \neq j$ we have $q \rho_{k} \in \mathbb{Z}_{+}$, and moreover $q\left(1+f_{j}(\tau)\right) \in \mathbb{Z}_{+}$(we stress on the fact that $j$ is a fixed index).

If we denote by $\left(\tau_{j}, \rho_{j}\right)$ the vertices of the polytope described by the conditions $(\mathbf{1})$ and $(\mathbf{2})$ above, then the affine coordinates of a point $(\tau, \rho)$ can be written as

$$
(\tau, \rho)=\sum_{p} \lambda^{p}\left(\tau_{p}, \rho_{p}\right)
$$

Now if $q$ is a rational number satisfying $(\mathbf{3})$, in general it does not follows that for all $p$ we have

$$
q \lambda^{p} \in \frac{1}{d_{0}} \mathbb{Z}
$$

where $d_{0}$ is some fixed integer.

In conclusion, the restricted algebra associated to $\mathrm{R}_{j}$ is far too general in order to apply the results which are proved in [19]-in order to use them, the relation ( $\dagger \dagger)$ is needed.

The approach we have presented in this survey avoids exactly this rationality issue, due to the fact that we only perform a finite number of iterations, and each iteration needs only a finite number of arithmetic conditions for the order of truncation of the initial algebra. 


\section{References}

[1] Ambro, F.: Restrictions of log canonical algebras of general type ;arXiv:math/0510212.

[2] Birkar, C., Cascini, P., Hacon, C., McKernan, J.: Existence of minimal models for varieties of log general type; on the web.

[3] Birkar, C., Păun, M.: Minimal models, flips and finite generation : a tribute to V.V. SHOKUROV and Y.-T. SIU arXiv:0904.2936.

[4] Boucksom, S. Divisorial Zariski decompositions on compact complex manifolds ; Ann. Sci. Ecole Norm. Sup. (4) 37 (2004), no. 1, 45-76.

[5] Boucksom, S., Eyssidieux, Ph., Guedj, V., Zeriahi, A. Monge-Ampre equations in big cohomology classes ; arXiv:0812.3674.

[6] Claudon, B.: Invariance for multiples of the twisted canonical bundle ; math.AG/0511736, Ann. Inst. Fourier (Grenoble) 57 (2007), no. 1, 289-300.

[7] Demailly, J.-P., Peternell, Th., Schneider, M. : Pseudoeffective line bundles on compact Kähler manifolds ; Internat. J. Math. 12 (2001), no. 6.

[8] Demailly, J.-P.: Kähler manifolds and transcendental techniques in algebraic geometry ; Plenary talk and Proceedings of the Internat. Congress of Math., Madrid (2006), 34p, volume I.

[9] Demailly, J.-P.:Analytic approach of the minimal model program and of the abundance conjectures, ; Park City Math Institute Lecture Notes, July 2008.

[10] Druel, S.: Existence de modèles minimaux pour les variétés de type général ; Exposé 982, Séminaire Bourbaki, 2007/08.

[11] Ein, L., Lazarsfeld, R., Mustaţă, M., Nakamaye, M., Popa, M. :Asymptotic invariants of base loci ; Ann. Inst. Fourier (Grenoble) 56 (2006), no. 6, 1701-1734.

[12] Ein, L., Popa, M.: Adjoint ideals and extension theorems ; preprint in preparation ; june 2007.

[13] Hacon, C., McKernan, J.: Boundedness of pluricanonical maps of varieties of general type ; Invent. Math. Volume 166, Number 1 / October, 2006, 1-25.

[14] Hacon, C., McKernan, J.: On the existence of flips ; math.AG/0507597.

[15] Kawamata, Y.: Finite generation of a canonical ring ; arXiv:0804.3151.

[16] Kawamata, Y.: Remarks on the cone of divisors ; arXiv:0909.3621v1.

[17] Kollár, J., Mori, S.: Birational geometry of algebraic varieties. Cambridge University Press, Cambridge, 1998.

[18] Lazarsfeld, R.: Positivity in Algebraic Geometry ; Springer, Ergebnisse der Mathematik und ihrer Grenzgebiete.

[19] Lazić, V.: Adjoint rings are finitely generated; arXiv:0905.2707.

[20] Nakayama, N.:Zariski decomposition and abundance ; MSJ Memoirs 14, Tokyo (2004).

[21] Păun, M.: Siu's Invariance of Plurigenera: a One-Tower Proof ; preprint IECN (2005), J. Differential Geom. 76 (2007), no. 3, 485493.

[22] Păun, M.: Relative critical exponents, non-vanishing and metrics with minimal singularities arXiv:0807.3109.

[23] Shokurov, V.: A non-vanishing theorem ; Izv. Akad. Nauk SSSR (49) 1985.

[24] Shokurov, V.: 3-fold log models. Algebraic geometry 4, J. Math. Sci. 81 (1996), no. 3, 26672699.

[25] Siu, Y.-T.: Analyticity of sets associated to Lelong numbers and the extension of closed positive currents Invent. Math. 27 (1974), 53-156.

[26] Siu, Y.-T.: Invariance of Plurigenera ; Inv. Math., 134 (1998), 661-673.

[27] Siu, Y.-T.: Extension of twisted pluricanonical sections with plurisubharmonic weight and invariance of semipositively twisted plurigenera for manifolds not necessarily of general type ; Complex geometry (Göttingen, 2000), 223-277, Springer, Berlin, 2002.

[28] Siu, Y.-T.: A General Non-Vanishing Theorem and an Analytic Proof of the Finite Generation of the Canonical Ring ; arXiv:math/0610740.

[29] Siu, Y.-T.: Finite Generation of Canonical Ring by Analytic Method ; arXiv:0803.2454.

[30] Siu, Y.-T.: Techniques for the Analytic Proof of the Finite Generation of the Canonical Ring ; arXiv:0811.1211. 
[31] Takayama, S: On the Invariance and Lower Semi-Continuity of Plurigenera of Algebraic Varieties ; J. Algebraic Geom. 16 (2007), no. 1, 1-18.

[32] Takayama, S: Pluricanonical systems on algebraic varieties of general type ;Invent. Math. Volume 165, Number 3 / September, 2005, 551-587.

[33] Tsuji, H.: Extension of log pluricanonical forms from subvarieties ; math.CV/0511342 .

[34] Varolin, D.: A Takayama-type extension theorem ; math.CV/0607323, to appear in Comp. Math.

(version of October, 2009, printed on December 16, 2009)

Mihai Păun, paun@iecn.u-nancy.fr 